\title{
Localization for random perturbations of periodic Schrödinger operators
}

\author{
Werner KIRSCH ${ }^{1}$, Peter STOLLMANN ${ }^{2}$ and Günter STOLZ ${ }^{3}$ \\ 1 Institut für Mathematik, R.uhr-Universität Bochum \\ D-44780 Bochum, Germany \\ ${ }^{2}$ Fachbereich Mathematik, Johann Wolfgang Goethe-Universität \\ D-60054 Frankfurt am Main, Germany \\ 3 University of Alabama at Birmingham, Department of Mathematics \\ Birmingham, Alabama 35294, USA
}

Received for ROSF, July 1, 1997

\begin{abstract}
We prove localization for Anderson-type random perturbations of periodic Schrödinger operators on $\mathrm{R}^{d}$ near the band edges.

General, possibly unbounded, single site potentials of fixed sign and compact support are allowed in the random perturbation. The proof is based on the following methods:

(i) A study of the band shift of periodic Schrödinger operators under linearly coupled periodic perturbations.

(ii) A proof of the Wegner estimate using properties of the spatial distribution of eigenfunctions of finite box hamiltonians.

(iii) An improved multiscale method together with a result of de Branges on the existence of limiting values for resolvents in the upper half plane, allowing for rather weak disorder assumptions on the random potential.
\end{abstract}

(iv) Results from the theory of generalized eigenfunctions and spectral averaging.

The paper aims at high accessibility in providing details for all the main steps in the proof.

\section{INTRODUCTION, THE MODEL AND THE RESULTS}

One of the most interesting issues concerning mathematical models of solid state physics is localization. Mathematically it is most commonly described by the occurence of pure point spectrum with rapidly decaying eigenfunctions for random operators in a certain energy region (see, however [6]). More precisely, consider a periodic Schrödinger operator $H_{\text {per }}=-\Delta+V_{\text {per }}$ describing the one-electron approximation of a perfect crystal. Then $H_{\text {per }}$ has purely absolutely continuous spectrum consisting of closed intervals, called bands, under some mild assumptions on $V_{\text {per. }}$. Adding an Anderson type random potential $V_{\omega}$ to $H_{\text {per }}$ we get an operator $H_{\omega}$ which corresponds to the crystal with impurities distributed according to $V_{\omega}$. This perturbation, even if it is very small with respect to $H_{p e r}$, causes a drastic change of the spectral picture: near the band edges of $\sigma\left(H_{\omega}\right)$ the operator exhibits localization in the above sense. This is the

\footnotetext{
2 Research supported by DFG and F.PSCoR of Alabama

3 Research supported by NSF-Grant DMS-9401417
} 
content of Theorem 1.1 below, where we need a certain "disorder regime", condition (1.2) below. Results which are closely related to Theorem 1.1 have recently been given by Barbaroux, Combes and Hislop in [1], and by Figotin and Klein in [10] and [11]. Figotin and Klein also proved a discrete version of Theorem 1.1 in [9].

For the bottom of the spectrum we present a result, Theorem 1.2, in which no additional disorder assumption is needed. Thus it partly improves upon the results of Combes and Hislop, [2], and Klopp, [19]; see however a result of Kotani and Simon, [21], who treat a special model without disorder assumption. The difference between general band edges and the bottom of the spectrum lies in the fact that for the latter it is known that the the density of states exhibits Lifshitz tails, see [17]. This idea has been used before to prove localization in [19].

Let us now briefly describe the organization of the paper: In the rest of this section we introduce the model we are working with, and state the main results. We also discuss some basic technical facts, in particular we characterize the almost sure spectrum of $H_{\omega}$.

Sections 2 to 7 are devoted to build up the machinery needed for the proofs of Theorems 1.1 and 1.2, which are finally completed in Section 8. The center of this machinery is a multiscale analysis, a technique which has been used in most of the proofs of multidimensional localization. We use a version which is close to the treatment by Combes and Hislop in [2], which itself is based on a discrete multiscale method used by von Dreifus and Klein [7], see also [29].

The two main ingredients for the multiscale analysis, a Wegner estimate and the initial length scale estimate, are presented and proven in Sections 3 and 4 . Their use has also become standard meanwhile, but two specific features of our presentation should be pointed out: First, we have a rather short and direct method to prove a Wegner estimate which uses an idea of one of us from [14]. Second, we aim to include single site potentials of small support (see Assumption A below) under minimal disorder assumptions. In particular, we do not assume 'large disorder' in the sense that the random coupling constansts $q_{k}$ to be introduced below have a distribution with large support. This needs some non-trivial preparation in Section 2, where we show that the bands of a periodic Schrödinger operator are shifted linearly by a linearly coupled periodic perturbation.

Sections 5 and 6 present the multiscale analysis with complete proofs. Some recent ideas have been added with the effect of streamlined proofs and somewhat stronger results. One improvement arises from an improved Combes-Thomas method due to [1]. We use it in the proof of Lemma 5.5, which is given in an Appendix. Another idea is to use a basic functional analytic result of de Branges [5] on boundary values of operator valued analytic functions in our proof of exponential decay of the resolvent in Section 6. This idea has been used in a similar context in [33].

The conclusion of the proof of our main results in Section 8 is based on two additional basic methods from spectral theory: spectral averaging and expansions in generalized eigenfunctions. We state what we need from these theories in Section 7 . Note that generalized eigenfunctions have been used in other works on localization, cf. [7], and can be seen as an alternative to a method based on Fredholm theory and the de la Vallée-Poussin theorem used in [2]. We end our paper with some conclusive remarks concerning some readily available extensions and generalizations of the results presented here and a discussion on the relation of our results to the works [1], [10] and [11]. We did not include the generalizations in the first place, since it is definitely an aim of this article to invite non-specialists to the subject of localization. Thus we have not striven 
for overwhelming generality but rather tried to present the main ideas as clearly as possible in their simplest form.

We now present the model we are dealing with. Throughout we rest on the following assumptions:

A. Let $p=2$ if $d \leq 3$, and $p>d / 2$ if $d \geq 4$ and let $V_{\text {per }} \in L_{\text {loc }}^{p}$ be a $\mathrm{Z}^{d}$-periodic real potential, $H_{p e r}=-\Delta+V_{p e r}$. As a random potential consider an Anderson type potential of the form

$$
V_{\omega}(x)=\sum_{k \in Z^{d}} q_{k}(\omega) \cdot f(x-k),
$$

where the single site potential $f \in L^{p}$ and the $q_{k}$ satisfy:

A.1 For some $0<s \leq 1: f \geq \chi_{\Lambda_{s}(0)}$, where $\Lambda_{r}(x)$ denotes an open cube of sidelength $r$ centered at $x$.

A.2 The support of $f$ is contained in $\Lambda_{1}(0)$.

A.3 The $q_{k}$ are i.i.d. random variables with common density $g \in L^{\infty}$.

A.4 The support of $g$ is a compact interval $\left[q_{-}, q_{+}\right]$.

We denote by $\Omega$ the product $\left[q_{-}, q_{+}\right]^{\mathbf{Z}^{d}}$ and by $\mathbf{P}$ the product measure $\bigotimes_{k \in Z^{d}} g\left(q_{k}\right) d q_{k}$. Thus, for $\omega \in \Omega$ we have $q_{k}(\omega)=\omega_{k}$.

Clearly, in A.1 it would suffice to have a lower estimate by some positive number on some smaller cube. Note that the support of $f$ can be arbitrarily small. A.2 can be weakened to only assuming that $f$ is compactly supported. The necessary changes in the proof are discussed in Section 9. Also, A.4 is mainly needed to have the following formula for the spectrum of $H_{\omega}=H_{p e r}+V_{\omega}$ :

$$
\sigma\left(H_{\omega}\right)=\bigcup_{q \in\left[q_{-}, q_{+}\right]} \sigma\left(H_{p e r}+q \cdot \sum_{k \in Z^{d}} f(\cdot-k)\right)=: \Sigma \quad \mathbf{P}-a . s .
$$

where the fact that the spectrum is deterministic is already clear from ergodicity. For the case $V_{\text {per }}=0$ this formula is well known, cf. [16]. We defer the easy proof of (1.1) to the end of this section. Note that $\Sigma$ has band structure, since the operators occuring in the formula for $\Sigma$ are all periodic and their band edges depend continuously on $q$, see the discussion of basic properties of periodic operators below. Moreover, the boundary points of $\Sigma$ correspond to band edges of either $H_{p e r}+q_{+} \cdot \sum_{k \in \mathrm{Z}^{d}} f(\cdot-k)$ or $H_{\text {per }}+q_{-} \cdot \sum_{k \in Z^{d}} f(\cdot-k)$. Thus they "correspond" to the rare events that almost all the coupling constants are near $q_{+}$or $q_{-}$. This is the reason for the name fluctuation boundaries and it is near these boundaries where one expects localization. This quite vague picture can be made precise on the level of the corresponding operators restricted to finite boxes (see Section 4 below) and is the basis for the proof of localization. Finally, note that existing gaps in $\sigma\left(H_{\text {per }}\right)$ may be filled-in for $\Sigma$, but it follows again from continuity that $\Sigma$ will have non-trivial gaps if $\sigma\left(H_{p e r}\right)$ has gaps and $q_{+}$is close to $q_{-}$.

Here are our main results:

THFORFM 1.1. Assume that there exists $\tau>d / 2$ such that $g$ satisfies

$$
\int_{q_{-}}^{q_{-}+h} g(s) \mathrm{d} s \leq h^{\tau} \text { and } \int_{q_{+}-h}^{q_{+}} g(s) \mathrm{d} s \leq h^{\tau} \text { for small } h>0 .
$$

Then in a neighborhood of $\partial \Sigma$ the spectrum of $H_{\omega}$ is pure point $\mathbf{P}$-a.s. with exponentially decaying eigenfunctions. 
It will be clear from the proof that if we require the decay of $g$ only near $q_{+}$or $q_{-}$we get localization, but only at the upper or lower band edges respectively. Using estimates from the theory of Lifshitz tails we get the following improvement for the bottom of the spectrum.

THFORFM 1.2. In a neighborhood of inf $\Sigma$ the spectrum of $H_{\omega}$ is pure point $\mathbf{P}$-a.s. with exponentially decaying eigenfunctions.

In his recent preprint [20] Klopp established the existence of Lifshitz tails at internal band edges provided the unperturbed periodic operator satisfies a nondegeneracy condition. Under this condition the assumption (1.2) could be removed in Theorem 1.1.

The class of potentials used in this work is standard in the theory of periodic Schrödinger operators, c.f. [27, Ch. XIII]. Nevertheless, we now briefly discuss some of its properties, in particular, to point out the uniform dependence of operator bounds on several parameters.

Let $V$ be a potential on $\mathbf{R}^{d}$ which is locally uniformly in $L^{p}$, with $p$ as above. For a cube $\Lambda$ with integer sidelength $\ell$ and $\Theta \in[0,2 \pi)^{d}$ let $C_{\Theta}^{\infty}(\Lambda)$ be the $=C^{\infty}$-functions $\varphi$ on $\mathrm{R}^{d}$ which are $\Theta$-quasiperiodic w.r.t. $\Lambda,=$ i.e. $\varphi\left(x+\ell e_{k}\right)=\mathrm{e}^{i \Theta_{k}} \varphi(x), k=1, \ldots, d$.

The proof of $\Delta$-boundedness of $V$ with relative bound zero in [27, Theorem XIII.96], i.e. self-adjointness of $-\Delta+V$ on $L^{2}\left(\mathrm{R}^{d}\right)$, can easily be extended to show the following: For every $\varepsilon>0$ there is a $C(\varepsilon)$ such that

$$
\|V \varphi\|_{L^{2}(\Lambda)} \leq \varepsilon\|\Delta \varphi\|_{L^{2}(\Lambda)}+C(\varepsilon)\|\varphi\|_{L^{2}(\Lambda)}
$$

for every cube $\Lambda, \Theta \in[0,2 \pi)^{d}$ and $\varphi \in C_{\Theta}^{\infty}(\Lambda)$. Moreover, the proof shows that $C(\varepsilon)$ can be chosen uniformly w.r.t. a family of potentials $V$ if $\sup _{x}\|V\|_{L^{p}\left(\Lambda_{1}(x)\right)}$ is uniformly bounded in $V$. This applies to the family $\left\{V_{\text {per }}+V_{\omega} \mid \omega \in \Omega\right\}$ considered by us and will be used several times in the sequel. The operators $H_{\Lambda}^{\Theta}=(-\Delta+V)_{\Lambda}^{\Theta}$ on $L^{2}(\Lambda)$ are therefore self-adjoint with domain $W_{\Theta}^{2,2}(\Lambda)=\bar{C}_{\Theta}^{\infty}(\Lambda) W^{2,2}$. For $\Theta=0$, i.e. periodic boundary conditions, we just write $H_{\Lambda}=(-\Delta+V)_{\Lambda}$.

If $V \in L_{l o c}^{p}$ with $p$ as above is $Z^{d}$-periodic, then recall from [27, Ch.XIII.16] that $\sigma(-\Delta+V)=\bigcup_{n} \bigcup_{\Theta \in[0,2 \pi)^{d}} E_{n}(\Theta)$, where $E_{n}(\Theta)$ is the $n$-th eigenvalue of $(-\Delta+$ $V)_{\Lambda_{1}(0)}^{\Theta} . E_{n}(\Theta)$ is continuous in $\Theta$, which can be seen from the min-max characterization of eigenvalues and the quasi momentum representation $U_{\Theta}^{-1}(-\Delta+V)_{\Lambda_{1}(0)}^{\ominus} U_{\Theta}=(-\Delta+$ $\left.V+|\Theta|^{2}+\mathrm{i} \Theta \cdot \nabla\right)_{\Lambda_{1}(0)}$ with the unitary operator $\left(U_{\Theta} f\right)(x)=\mathrm{e}^{-\mathrm{i} \Theta \cdot x} f(x)$ in $L^{2}\left(\Lambda_{1}(0)\right)$. If $V=V_{1}+\lambda V_{2}$, where $V_{1}$ and $V_{2}$ are both $\mathrm{Z}^{d}$-periodic, then similar considerations show that the "band edges" $\min _{\Theta} E_{n}(\Theta, \lambda)$ and $\max _{\Theta} E_{n}(\Theta, \lambda)$ depend continuously on $\lambda$.

As promised, we now sketch the

Proof of (1.1). "C": By standard convergence results we have that

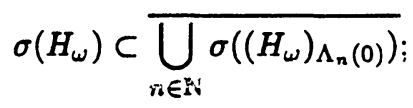

on the other hand, for fixed $n \in \mathbf{N}$, it follows by min-max that

$$
\sigma\left(\left(H_{\omega}\right)_{\Lambda_{n}(0)}\right) \subset \Sigma
$$

since the $k$-th eigenvalue of $\left(H_{\omega}\right)_{\Lambda_{n}(0)}$ lies between the $k$-th eigenvalue of $\left(-\Delta+V_{\text {per }}+\right.$ $\left.q_{-} \cdot \sum_{k} f(\cdot-k)\right)_{\Lambda_{n}(0)}$ and the $k$-th eigenvalue of $\left(-\Delta+V_{p e r}+q_{+} \cdot \sum_{k} f(\cdot-k)\right)_{\Lambda_{n}(0)}$. 
"ᄀ": Given a $q \in\left[q_{-}, q_{+}\right]$and an $E \in \sigma\left(-\Delta+V_{\text {per }}+q \cdot \sum_{k} f(\cdot-k)\right)$ we find a singular sequence $\phi_{n}$ in $C_{c}^{\infty}$ for $E$. Let $l_{n} \in \mathrm{N}$ be such that $\operatorname{supp}\left(\phi_{n}\right) \subset \Lambda_{l_{n}}(0)$, and for $x \in \mathrm{Z}^{d}$ consider

$$
\Omega_{n}(x):=\left\{\omega \in \Omega ; q_{k}(\omega) \in\left[q-\frac{1}{n}, q+\frac{1}{n}\right] \text { for all } k \in \Lambda_{l_{n}}(x)\right\} .
$$

Then $\mathbf{P}\left(\Omega_{n}(x)\right)$ is independent of $x$ and strictly positive. Also, if $\Lambda_{\ell_{n}}(x) \cap \Lambda_{\ell_{n}}(y)=\emptyset$, then $\Omega_{n}(x)$ and $\Omega_{n}(y)$ are independent. Thus, for a.e. $\omega \in \Omega$ there exists an $x_{n}(\omega)$ such that $\omega \in \Omega_{n}\left(x_{n}(\omega)\right)$. By countability it follows that for a.e. $\omega$ this holds for all $n$. This implies

$$
\left\|\left(H_{\omega}-E\right) \phi_{n}\left(\cdot-x_{n}(\omega)\right)\right\| \leq \frac{1}{n}\left\|\sum_{k} f(\cdot-k) \phi_{n}\right\|+\|\left(H_{p e r}+q \sum_{k} f(\cdot-k) \phi_{n} \| \rightarrow 0\right.
$$

as $n \rightarrow \infty$, where a relative boundedness argument has to be used on $\left\|\sum_{k}=f(\cdot-k) \phi_{n}\right\|$. Thus $\phi_{n}\left(\cdot-x_{n}(\omega)\right)$ is a singular sequence for $E$ to $H_{\omega}$. This concludes the proof of (1.1).

In the context of random perturbations of diffusion matrices one can give a similar description of the spectrum; this was done by one of us in [3]. As usual we use the letter $C$ for a constant that may be different in different equations or inequalities; moreover we will often leave the dependence upon the space dimension $d$ implicit.

\section{PERTURBATION OF BANDS}

In this section we provide the tools for our proof of the Wegner estimate and the initial length scale estimates. As the title indicates it is mainly about how the bands of a periodic Schrödinger operator behave under perturbation by certain periodic potentials. The following result is a step in this direction, since the quantity estimated from below in (2.1) will show up as a derivative of eigenvalues.

PROPOSITION 2.1. Let $V_{0}$ and $V$ be locally uniformly in $L^{p}$ with $p$ as in Assumption $A$ and denote by $H_{\Lambda}^{0}$ and $H_{\Lambda}$ the corresponding Schrödinger operators with periodic boundary conditions on the cube $\Lambda \subset \mathrm{R}^{d}$. Let $F \subset \Lambda$ such that $\operatorname{dist}\left(\left\{x \in \Lambda ; V_{0}(x) \neq\right.\right.$ $\left.V(x)\}, F \cup \Lambda^{c}\right)=: \vartheta>0, \chi:=1-\chi_{F}$. Then there is a constant $C=C(\vartheta)$ such that for every eigenfunction $f$ of $H$ with eigenvalue $\mu \in \rho\left(H_{\Lambda}^{0}\right)$ we have

$$
\|f\| \leq\left[1+C\left(\left\|\left(H_{\Lambda}^{0}-\mu\right)^{-1}\right\|+\left\|\left(H_{\Lambda}^{0}-\mu\right)^{-1} \nabla\right\|\right)\right]\|\chi f\| .
$$

Proof. Choose a $C^{2}$-cut-off function $0 \leq \phi \leq 1$ which vanishes on a neighborhood of $F$ and $\partial \Lambda$ and is 1 on a neighborhood of $\left\{x ; V_{0}(x) \neq V(x)\right\}$. We can choose it in such a way that the supremum norms of its derivatives are bounded in terms of $\vartheta$. Note that with $f$ also $(1-\phi) f \in D\left(H_{\Lambda}^{0}\right)$ by the assumptions on $\phi$.

$$
\begin{aligned}
\|(1-\phi) f\| & =\left\|\left(H_{\Lambda}^{0}-\mu\right)^{-1}\left(H_{\Lambda}^{0}-\mu\right)(1-\phi) f\right\| \\
& =\left\|\left(H_{\Lambda}^{0}-\mu\right)^{-1}\left[(1-\phi)\left(H_{\Lambda}-\mu\right) f+(\Delta(1-\phi)) f-2 \nabla[\nabla(1-\phi)] f\right]\right\| \\
& =\left\|\left(H_{\Lambda}^{0}-\mu\right)^{-1}[(\Delta(1-\phi)) f-2 \nabla[\nabla(1-\phi)]=f]\right\|,
\end{aligned}
$$


where we used that $f$ is an eigenfunction of $H$. Since $\Delta(1-\phi)$ and $\nabla(1-\phi)$ are supported on $F$ we can estimate

$$
\leq C(\vartheta)\left(\left\|\left(H_{\Lambda}^{0}-\mu\right)^{-1}\right\|+=\left\|\left(H_{\Lambda}^{0}-\mu\right)^{-1} \nabla\right\|\right)\|\chi f\|
$$

which proves the assertion by noting that $\|\phi f\| \leq\|\chi f\|$.

The following result is of interest beyond its application in Section 4.

THFORFM 2.2. Let $V, W \in L_{\text {loc }}^{p}$ be $\mathrm{Z}^{d}$-periodic and $p$ as in Assumption A. Assume that $W \geq 0$ is such that the interior of $\{x: W(x) \geq \alpha\}$ is non-empty for some $\alpha>0$. Let $H=-\Delta+V, H(\lambda)=H+\lambda W$ and denote by $a(\lambda) \in \partial \sigma(H(\lambda))$ a band edge varying continuously with $\lambda \in\left(-\lambda_{0}, \lambda_{0}\right)$. Then there exists $\lambda_{1}>0$ and $C>0$ such that

$$
|a(\lambda)-a(0)| \geq C|\lambda| \text { for }|\lambda| \leq \lambda_{1}
$$

Before stating the proof let us note that by the remarks in Section 1 for each band edge $a \in \partial \sigma(H)$ we find a continuous function $a(\cdot)$ as indicated.

Proof. By [27, XIII.16] we know that for some $n \in \mathrm{N}$ and all $\lambda \in\left(-\lambda_{0}, \lambda_{0}\right)$ we have

$$
a(\lambda)=E_{n}\left(H_{\Theta(\lambda)}(\lambda)\right)
$$

for some $\Theta(\lambda) \in[0,2 \pi)^{d}$, where $E_{n}(T)$ denotes the $n$-th eigenvalue of the operator $T$ and $H_{\Theta(\lambda)}(\lambda)$ is short for $(H(\lambda))_{\Lambda_{1}(0)}^{\Theta(\lambda)}$ (see Section 1). By monotonicity, we can assume without restriction that $W \in L^{\infty}$. We can also assume that the interior of $\{x ; W(x) \geq \alpha\}$ contains 0 .

Consider first the case where $a(\cdot)$ is an upper band edge and $\lambda>0$. Choose an orthonormal basis $\left(f_{k}\right)$ of eigenfunctions of $H_{\Theta(0)}$ with eigenvalues $E_{k}(0)$, i.e. for $\lambda=0$. The min-max principle gives

$$
E_{n}\left(H_{\Theta(0)}(\lambda)\right) \geq \inf _{g \perp\left\{f_{1}, \ldots, f_{n-1}\right\},\|g\|=1}((H+\lambda W) g \mid g) .
$$

Since the corresponding operator has compact resolvent, only finitely many $E_{k}(0)$ can equal $E_{n}(0)$, say at most those with $k \leq N$. We write $P$ and $Q$ for the orthogonal projections onto the subspaces spanned by $f_{n}, \ldots, f_{N}$ and $f_{N+1}, \ldots$ respectively and have

$$
\cdots \geq \inf \left[E_{n}(0)\|P g\|^{2}+E_{N+1}(0)\|Q g\|^{2}+\lambda(W g \mid g)\right] .
$$

Assume that the inf equals $E_{n}(0)$. Then there exists a normalized sequence $\left(g_{k}\right)$ in $\left\{f_{n}, \ldots, f_{N}\right\}^{\perp}$ such that $\left\|Q g_{k}\right\| \rightarrow 0$ and $\left(W g_{k} \mid g_{k}\right) \rightarrow 0$. Therefore, also $\left(W P g_{k} \mid P g_{k}\right) \rightarrow$ 0 . Since $P$ has finite dimensional range, there is an accumulation point $g=P g$ of the $g_{k}$ with $(W g \mid g)=0$. Since $W$ is strictly positive on some open set, this would contradict the unique continuation result from [12], since $g$ is an eigenfunction of $H_{\Theta(0)}$ with eigenvalue $E_{n}(0)$. (See [28] for more recent developments concerning unique continuation.) Thus $a(\lambda) \geq E_{n}\left(H_{\Theta(0)}(\lambda)\right)>E_{n}(0)=a(0)$.

From this we also get that $a(\lambda)<a(0)$ for an upper band edge and $\lambda<0$ (treat $H(\lambda)$ as the unperturbed operator). Similarly, we can prove that in case of a lower band edge $a(\cdot)$ and $\lambda>0$ we have that $a(\lambda)>a(0)$ (here we use an argument as in the first case, with $a(\lambda)=E_{n}\left(H_{\Theta(\lambda)}(\lambda)\right),\left(f_{k}\right)$ an orthonormal basis of eigenfunctions for 
$\left.H_{\Theta(\lambda)}(0)\right)$. Again it follows by reversing the roles of $H(\lambda)$ and $H(0)$ that $a(\lambda)<a(0)$ for small $\lambda<0$. In order to show the assertion of the theorem we use kind of a boot-strap argument and apply Proposition 2.1:

Consider now a lower band edge and $\lambda<0$. Fix $\alpha>0$ such that $\{x ; W(x) \geq \alpha\}$ contains the cube $\Lambda_{R}(0)$ for some $R>0$. For later use we also choose $R \leq 1$. By periodicity of $W$ we know that

$$
W \geq \alpha \text { on } \bigcup_{j \in Z^{d}} \Lambda_{R}(j) .
$$

Let $U$ be an open interval containing $a=a(0)$ such that $a=\inf \sigma(H) \cap U$. Let $0<\lambda_{2}$ be so small that, for $\lambda \in\left[-\lambda_{2}, 0\right]$, we have

$$
a(\lambda)=\inf \sigma(H(\lambda)) \cap U .
$$

As an auxiliary potential consider the characteristic function of

$$
\bigcup_{j \in Z^{d}} \Lambda_{r}(j)
$$

for some $0<r<R$, denoted by $W_{1}$. Then $b(t):=\inf \sigma\left(H+t W_{1}\right) \cap U$ is a band edge of $H+t W_{1}$ for $t$ small enough; by what we saw above, $b(t)>a$ for $t>0$ small and fixed. By continuity in $\lambda$ we find $0<\lambda_{1}<\lambda_{2}$, a compact neighborhood $J$ of $a$ and $\delta>0$ such that

$$
\operatorname{dist}\left(\sigma\left(H(\lambda)+t W_{1}\right), J\right) \geq \delta \text { for } \lambda \in\left[-\lambda_{1}, 0\right]
$$

and

$$
a(\lambda) \in J \text { for } \lambda \in\left[-\lambda_{1}, 0\right] \text {. }
$$

By standard theory of periodic Schrödinger operators we know that

$$
\sigma(H(\lambda))=\overline{\bigcup_{k \in \mathbb{N}} \sigma\left(H_{\Lambda_{k}(0)}(\lambda)\right)}
$$

so that

$$
a(\lambda)=\inf \left(\left\{E_{n}\left(H_{\Lambda_{k}(0)}(\lambda)\right) ; k, n \in \mathrm{N}\right\} \cap . J\right) .
$$

We will show below that there exists $C>0$, such that

$$
\frac{\mathrm{d}^{+}}{\mathrm{d} \lambda} E_{n}\left(H_{\Lambda_{h}(0)}(\lambda)\right) \geq C \text { and } \frac{\mathrm{d}^{-}}{\mathrm{d} \lambda} E_{n}\left(H_{\Lambda_{h}(0)}(\lambda)\right) \geq C
$$

for all $\lambda \in\left[-\lambda_{1}, 0\right]$ and $E_{n}\left(H_{\Lambda_{h}(0)}(\lambda)\right) \in J$ where $\frac{\mathrm{d}^{+/-}}{\mathrm{d} \lambda}$ denotes the right and left derivatives with respect to $\lambda$.

Let us first note that (2.6) will imply the desired estimate

$$
a(\lambda)-a(0) \leq C \lambda \text { for } \lambda \in\left[-\lambda_{1}, 0\right] .
$$

In fact, by (2.5) we find for $\varepsilon>0$ natural numbers $n, k$ such that

$$
E_{n}\left(H_{\Lambda_{k}(0)}(0)\right) \leq a(0)+\varepsilon
$$


Together with (2.6) this gives

$$
\begin{aligned}
a(\lambda) & \leq E_{n}\left(H_{\Lambda_{h}(0)}(\lambda)\right) \\
& =E_{n}\left(H_{\Lambda_{h}(0)}(0)\right)-\int_{\lambda}^{0} \frac{\mathrm{d}}{\mathrm{d} \lambda} E_{n}\left(H_{\Lambda_{h}(0)}(\lambda)\right) \mathrm{d} \lambda \\
& \leq a(0)+\varepsilon+C \lambda .
\end{aligned}
$$

To verify (2.6) we use Proposition 2.1. The set $J$ has already been defined. Let

$$
F:=\left(\bigcup_{j \in \mathrm{Z}^{d}} \Lambda_{R}(j)\right)^{c}
$$

so that

$$
W \geq \alpha \chi
$$

for $\chi=1-\chi_{F}$ and the assumption

$$
\operatorname{dist}\left(F \cup \Lambda_{k}(0)^{c},\left\{V+\lambda W+t W_{1} \neq V+\lambda W\right\}\right) \geq \frac{R-r}{2}
$$

holds (use $R \leq 1$ ). Thus we can apply Proposition 2.1 with the choice $H_{\Lambda}=H_{\Lambda_{k}(0)}(\lambda)$, $H_{\Lambda}^{0}=H_{\Lambda}+t W_{1}$, and $\mu=E \in J$. (2.4) and an argument using relative boundedness show that the constant in front of $\|\chi f\|$ in (2.1) is uniformly bounded in $\lambda \in\left[-\lambda_{1}, 0\right]$, $k$ and $E \in J$. This implies that every normalized eigenfunction $f$ of $H_{\Lambda_{k}(0)}(\lambda)$ with eigenvalue $E \in J$ satisfies

$$
\|\chi f\|^{2} \geq C \text {. }
$$

An application of the Feynman-Hellmann theorem (see [34], p.151) gives that for every normalized eigenfunction $f$ of $H_{\Lambda_{k}(0)}(\lambda)$ with eigenvalue $E_{n}\left(H_{\Lambda_{k}(0)}(\lambda)\right)$ we have

$$
\frac{\mathrm{d}}{\mathrm{d} \lambda} E_{n}\left(H_{\Lambda_{k}(0)}(\lambda)\right)=(W f \mid f) \geq \alpha\|\chi f\|^{2}
$$

If $E_{n}\left(H_{\Lambda_{k}(0)}(0)\right)$ is degenerate equation (2.8) holds for an appropriate choice of the eigenfunction (see [13, VII, 3, Thm. 3.6]). Since eigenvalue crossings might appear, the equation only holds for right and left derivatives. This, together with the estimate from (2.7) implies (2.6). We use the same idea for a lower band edge and $\lambda>0$; here again, an auxiliary potential $-t W_{1}$ will shift the spectrum away from $a$. Similar considerations hold for upper band edges: (2.6) holds with sup replacing inf and we again can apply Proposition 2.1 after shifting the spectrum to the right by $t W_{1}$ if $\lambda<0$ and to the left by $-t W_{1}$ if $\lambda>0$.

\section{THE WEGNER ESTIMATE}

In this section we are concerned with an inequality concerning the box hamiltonians

$$
H_{\Lambda}(\omega)=H_{p e r}+V_{\omega} \text { in } L^{2}(\Lambda)
$$

where $\Lambda=\Lambda_{\ell}(i)$ with $\ell \in \mathrm{N}, i \in \mathrm{Z}^{d}$ and we impose periodic boundary conditions. Here $H_{\text {per }}+V_{\omega}$ is the random Schrödinger operator given by Assumption A of Section 1. The 
Wegner estimate expresses quantitatively that it is quite unlikely to hit an eigenvalue of $H_{\Lambda}(\omega)$ for fixed $\Lambda$, varying $\omega$.

THFORFM 3.1. Let $a \in \partial \Sigma$. Then there is an open interval $I$ containing $a$ and a $C_{W}$ such that for all intervals $J \subset I$ and all $\Lambda=\Lambda_{\ell}(i)$ with $\ell \in \mathrm{N}, i \in \mathrm{Z}^{d}$ we have:

$$
\mathbf{P}\left\{\sigma\left(H_{\Lambda}(\omega)\right) \cap J \neq \emptyset\right\} \leq C_{W}|J||\Lambda|^{2}
$$

Since the spectrum of $H_{\Lambda}(\omega)$ consists of eigenvalues of finite multiplicity it is clear that an analysis of the mobility of these eigenvalues under varying $\omega$ will be crucial for the proof of Theorem 3.1. The $\omega_{k}$ have absolutely continuous distribution. Thus it is no surprise that we will have to study the derivatives of eigenvalues with respect to a coupling constant in the potential, which, as we already saw in the preceding section, is related to estimates of eigenfunctions. In fact, to obtain Theorem 3.1 we use the proof of the Wegner estimate in [14] verbatim, relying on the following result. Recall that $s$ is one of the parameters of our model as defined in Assumption A.

PROPOSITION 3.2. Let $a \in \partial \Sigma$. Then there is an open interval $I$ containing $a$ and $C>0$ such that for all $\Lambda=\Lambda_{\ell}(i)$ with $\ell \in \mathrm{N}, i \in \mathrm{Z}^{d}$, all $\omega \in \Omega$ and all normalized eigenfunctions $f$ of $H_{\Lambda}(\omega)$ corresponding to an eigenvalue $\mu \in I$ we have:

$$
\sum_{k \in \Lambda} \int_{\Lambda_{0}(k)}|f(x)|^{2} \mathrm{~d} x \geq C .
$$

Proof. We want to apply Proposition 2.1. Thus for $H=H_{\Lambda}(\omega)$ we want to find a comparison operator $H_{\Lambda}^{0}(\omega)=-\Delta+V_{0, \omega}$ with the following property: For

$$
F=\left(\bigcup_{k \in Z^{d}} \Lambda_{s}(k)\right)^{c}
$$

we have

$$
\operatorname{dist}\left(\left\{V_{0, \omega} \neq V_{\text {per }}+V_{\omega}\right\}, F\right)=: \vartheta>0
$$

independently of $\omega$. Assume first that $a$ is a lower band edge. We set

$$
V_{0, \omega}=V_{p e r}+V_{\omega}\left(1-\chi_{r}\right)+\lambda \chi_{r} \sum_{k} f(\cdot-k)
$$

for some $\lambda \in\left(q_{-}, q_{+}\right)$and $0<r<s$, where

$$
\chi_{r}=\sum_{k \in Z^{d}} \chi_{\Lambda_{r}(k)} .
$$

Clearly, (3.3) is satisfied for each $\lambda, \omega, \Lambda$. Moreover, for some $I$ as asserted,

$$
\operatorname{dist}\left(I, \sigma\left(H_{\Lambda}^{0}(\omega)\right) \geq \delta>0\right.
$$

for all $\omega \in \Omega$ and $\Lambda$. To verify this we use Theorem 2.2 : 
Note that $a$ is a lower band edge of $H_{\text {per }}+q_{-} \cdot \sum_{k \in Z^{d}} f(\cdot-k)$. Consequently, for $\lambda$ as above the corresponding band edge $a(\lambda)$ of $H(\lambda):=H_{p e r}+q_{-} \cdot \sum_{k \in Z^{d}} f(\cdot-k)(1-$ $\left.\chi_{r}\right)+\lambda \cdot \sum_{k \in Z^{d}} f(\cdot-k) \chi_{r}$ satisfies

$$
a(\lambda)>a,
$$

since the difference is $\left(\lambda-q_{-}\right) \cdot \sum_{k \in Z^{d}} f(\cdot-k) \chi_{r}$ so that we can apply Theorem 2.2 . For the restriction $H_{\Lambda}(\lambda)$ of $H(\lambda)$ to the cube with periodic boundary conditions we have that

$$
\min \{a(\lambda)-a, \operatorname{dist}(a, \partial \Sigma \backslash\{a\})\} \leq \operatorname{dist}\left(\sigma\left(H_{\Lambda}(\lambda)\right), a\right) \leq \operatorname{dist}\left(\sigma\left(H_{\Lambda}^{0}(\omega), a\right) .\right.
$$

Here, the latter inequality follows from $q_{-} \leq q_{k}(\omega)$ by the min-max principle. Therefore, (3.4) follows for a suitable open interval $I$ containing $a$.

(3.4) implies that $\left\|\left(H_{\Lambda}^{0}(\omega)-\mu\right)^{-1}\right\|$ is uniformly bounded in $\mu \in I, \omega$ and $\Lambda$, and an argument using uniform relative bounds in $\omega$ shows the same for $\left\|\left(H_{\Lambda}^{0}(\omega)-\mu\right)^{-1} \nabla\right\|$. Thus we can apply Proposition 2.1 and get the asserted (3.2).

In the case of upper band edges, which are band edges of $H_{p e r}+q_{+} \cdot \sum_{k \in Z^{d}} f(\cdot-k)$ we add the same auxiliary potential to shift the band edge to the left (this follows from $\left.\lambda<q_{+}\right)$and use the same arguments as above, incl. Proposition 2.1.

\section{INITIAL LENGTH SCALE ESTIMATES}

In this section we will prove the initial length scale estimates which are necessary for multi-scale analysis. Let us first consider the case of band edges above the bottom of the spectrum and recall that in this case, i.e. the case of Theorem 1.1 we assume that the coupling constant density $g$ satisfies:

$$
\int_{q_{-}}^{q_{-}+h} g(s) \mathrm{d} s \leq h^{\tau} \text { and } \int_{q_{+}-h}^{q_{+}} g(s) \mathrm{d} s \leq h^{\tau} \text { for some } \tau>d / 2 \text { and small } h>0
$$

By standard Combes-Thomas estimates, the exponential decay rates for resolvents we are aiming at will be reduced to proving a lower bound on the distance of the spectrum of the box hamiltonians $H_{\Lambda}(\omega)$ to a given band edge $a$.

Proposition 4.1. Assume (4.1) and let $a \in \partial \Sigma$. Then for any $\xi \in(0,2 \tau-d)$ there is a $\beta>0$ and $\ell^{*}=\ell^{*}(\tau, \xi)$ such that

$$
\mathbf{P}\left\{\operatorname{dist}\left(\sigma\left(H_{\Lambda}(\omega)\right) \cap(a, \infty), a\right) \leq \ell^{\beta-2}\right\} \leq \ell^{-\xi} \quad \text { if } a \text { is a lower band edge }
$$

and

$$
\mathbf{P}\left\{\operatorname{dist}\left(\sigma\left(H_{\Lambda}(\omega)\right) \cap(-\infty, a), a\right) \leq \ell^{\beta-2}\right\} \leq \ell^{-\xi} \text { if } \text { a is an upper band edge }
$$

for $\Lambda=\Lambda_{\ell}(0)$ and $\ell \geq \ell^{*}$.

Proof. Assume that $a$ is a lower band edge. We use Theorem 2.2 with

$$
W=\sum_{k \in Z^{d}} f(\cdot-k) \text { and } V=V_{p e r}+q_{-} \sum_{k \in Z^{d}} f(\cdot-k)
$$


and choose $C$ and $\lambda_{1}$ as asserted there. For $0<h=C^{-1} \ell^{\beta-2}, \beta<2, \ell \in \mathbf{N}$ consider

$$
\Omega_{\ell, h}=\left\{\omega \in \Omega ; q_{k}(\omega) \geq q_{-}+h \text { for all } k \in \Lambda_{\ell}(0)\right\}
$$

Then

$$
\mathbf{P}\left(\Omega_{\ell, h}\right) \geq 1-|\Lambda| \cdot \int_{q_{-}}^{q_{-}+h} g(s) \mathrm{d} s \geq 1-|\Lambda| h^{\tau}=1-C^{-\tau} \ell^{d-\tau(2-\beta)} .
$$

Moreover, for $\omega \in \Omega_{\ell, h}$ we have by monotonicity that

$$
\begin{aligned}
\operatorname{dist}\left(\sigma\left(H_{\Lambda}(\omega)\right) \cap(a, \infty), a\right) & \geq \operatorname{dist}(\sigma(-\Delta+V+h \cdot W) \cap(a, \infty), a) \\
& \geq C \cdot h \\
& =\ell^{\beta-2} .
\end{aligned}
$$

Starting with $0<\xi<2 \tau-d$ we find $\beta>0$ such that $\xi<\tau(2-\beta)-d$, and these $\beta, \xi$ will do for large enough $\ell$.

For an upper band edge we use

$$
\Omega_{\ell, h}=\left\{\omega \in \Omega ; q_{k}(\omega) \leq q_{+}-h \text { for all } k \in \Lambda_{\ell}(0)\right\}
$$

and apply Theorem 2.2 in the same way as above. We now turn to the discussion of the infimum of the spectrum. Here we will use results from the theory of Lifshitz tails (see [23, Chapter IV], [15] for a general discussion) in order to arrive at the analog of Proposition 4.1 without a disorder assumption of the form (4.1).

Proposition 4.2. Let $a=\inf \Sigma$. Then for any $\xi>0$ and $\beta \in(0,2)$ there is an $\ell^{*}=\ell^{*}(\xi, \beta)$ such that

$$
\mathbf{P}\left\{\operatorname{dist}\left(\sigma\left(H_{\Lambda}(\omega)\right), a\right) \leq \ell^{\beta-2}\right\} \leq \ell^{-\xi}
$$

for $\Lambda=\Lambda_{\ell}(0)$ and $\ell \geq \ell^{*}$.

Proof. Let us first consider the case of reflection invariant potentials which is treated in [17]. Denoting the Schrödinger operator $-\Delta+V_{\text {per }}+V_{\omega}$ on $\Lambda$ with Neumann boundary conditions by $H_{\Lambda}^{N}(\omega)$ it is clear from the min-max principle (compare [8, Ch.6.3]) that the first eigenvalues obey

$$
E_{1}\left(H_{\Lambda}^{N}(\omega)\right) \leq E_{1}\left(H_{\Lambda}(\omega)\right),
$$

where the latter refers, as usual, to the operator with periodic boundary conditions. Hence it is enough to prove

$$
\mathbf{P}\left\{E_{1}\left(H_{\Lambda}^{N}(\omega)\right) \leq a+\ell^{\beta-1}\right\} \leq \ell^{-\xi} .
$$

To this effect infer from [17], p. 804 that

$$
\mathbf{P}\left\{E_{1}\left(H_{\Lambda}^{N}(\omega)\right) \leq a+\ell^{-2}\right\} \leq \exp \left(-K \ell^{d / 2}\right)
$$

for some $K>0$. This is on the one hand not enough distance to $a$ but on the other hand a much better decay in $\ell$, the sidelength of the cube. Consider the bigger cube $\Lambda_{L}$ as a union of $\left(\frac{L}{\ell}\right)^{d}$ cubes of sidelength $\ell \in N$. Using the fact that

$$
H_{\Lambda_{L}}^{N}(\omega) \geq \bigoplus_{k \in(\ell Z)^{d} \cap \Lambda_{L}} H_{\Lambda_{\ell}(k)}^{N}(\omega)
$$


we get that

$$
E_{1}\left(H_{\Lambda_{L}}^{N}(\omega)\right) \geq \inf E_{1}\left(H_{\Lambda_{\ell}(k)}^{N}(\omega)\right)
$$

As there are $\left(\frac{L}{l}\right)^{d}$ smaller cubes in $\Lambda_{L}$ and the probability that a fixed one of them contributes an eigenvalue below $a+\ell^{-2}$ is given by (4.6) we obtain:

$$
\mathbf{P}\left\{E_{1}\left(H_{\Lambda_{L}}^{N}(\omega)\right) \leq a+\ell^{-2}\right\} \leq \exp \left(-K \ell^{d / 2}\right)\left(\frac{L}{\ell}\right)^{d}
$$

Choosing $\ell^{-2} \approx L^{\beta-2}$, i.e. $\ell \approx L^{1-\frac{\beta}{2}}$ integer, we arrive at an estimate

$$
\mathbf{P}\left\{E_{1}\left(H_{\Lambda_{L}}^{N}(\omega)\right) \leq a+L^{\beta-2}\right\} \leq \exp \left(-K L^{\alpha}\right) L^{\gamma}
$$

for some $\alpha>0$ and $\gamma$. Clearly this implies the desired polynomial decay in $L$.

In the general case we use the ideas from [22] in order to get the analog of (4.5). To this end consider boundary conditions of the type $H_{\Lambda}^{\chi}(\omega)$ which are defined in [22] by the help of a periodic strictly positive generalized eigenfunction $h$ of $H_{\text {per }}$. In [22] it is described how to modify the proof of [17] in order to show that these operators still satisfy (4.6) above. Moreover, from Proposition 1 in [22] it follows that (4.7) is valid. The final observation necessary to adopt the above proof to the general case is the fact that, as for the Neumann boundary conditions, we have

$$
H_{\Lambda}^{\chi}(\omega) \leq H_{\Lambda}(\omega)
$$

which is apparent from the special choice of $\chi$ in [22].

\section{MULTI-SCALE ANALYSIS}

Let $\delta_{0}>0$ be fixed, $|y|:=\max _{i}\left|y_{i}\right|$ for $y \in \mathbf{R}^{d}$ and dist $(\cdot, \cdot)$ be the corresponding distance function. For a cube $\Lambda_{\ell}(x)=\left\{y \in \mathrm{R}^{d}:|x-y|<\ell / 2\right\}$ with center $x \in$ $\mathrm{Z}^{d}$ and sidelength $\ell \in \mathrm{N}$ let $\varphi_{\ell, x} \in C_{0}^{\infty}\left(\Lambda_{l}(x)\right)$ be chosen such that $\varphi_{\ell, x}=0$ for $\operatorname{dist}\left(y, \partial \Lambda_{\ell}(x)\right) \leq \delta_{0}$, and $\varphi_{\ell, x}(y)=1$ for $\operatorname{dist}\left(y, \partial \Lambda_{\ell}(x)\right) \geq 2 \delta_{0}$. We can choose the $\varphi_{\ell, x}$ such that $\left|\partial_{i} \varphi_{\ell, x}\right|$ and $\left|\partial_{i} \partial_{j} \varphi_{\ell, x}\right|$ are uniformly bounded in $\ell$ and $x$ for all $i, j . \chi_{\ell, x}$ will denote the characteristic function of $\Lambda_{\ell, x}$ and (with $[\cdot, \cdot]$ denoting the commutator)

$$
W(\varphi):=[-\Delta, \varphi]
$$

for suitable functions $\varphi$. A tool which we use throughout the following sections is the geometric resolvent equation which is the following relation between resolvents of Hamiltonians restricted to different regions $\Lambda \subset \Lambda^{\prime}$. If $\phi_{\Lambda}$ denotes a $C^{2}$-function on $\Lambda$ it reads

$$
\phi_{\Lambda}\left(H_{\Lambda^{\prime}}-z\right)^{-1}=\left(H_{\Lambda}-z\right)^{-1} \phi_{\Lambda}+\left(H_{\Lambda}-z\right)^{-1} W\left(\phi_{\Lambda}\right)\left(H_{\Lambda^{\prime}}-z\right)^{-1}
$$

for $z$ in the resolvent set of $H_{\Lambda}$ and $H_{\Lambda^{\prime}}$.

For operators on cubes we introduce some more notation in order to facilitate the following calculations. With $V_{\text {per }}$ and $V=V_{\omega}$ as in Section 1 let

$$
H_{\ell, x}=-\Delta+V_{\text {per }}+V \text { on } L^{2}\left(\Lambda_{\ell}(x)\right)
$$


with periodic boundary conditions and $R_{\ell, x}(z)\left(H_{\ell, x}-z\right)^{-1}$. For $x=0$ the subscript $x$ will be dropped from the terminology defined above.

In the following we work with a fixed energy $E \in \mathrm{R}$.

Definition 5.1. A cube $\Lambda_{\ell}(x)$ is called $\gamma$-good for some $\gamma>0$ if

$$
\sup _{\varepsilon \neq 0}\left\|W\left(\varphi_{\ell, x}\right) R_{\ell, x}(E+\mathrm{i} \varepsilon) \chi_{\ell / 3, x}\right\| \leq \mathrm{e}^{-\gamma \ell} .
$$

Note that by independence the probability $\mathbf{P}\left(\Lambda_{\ell, x}\right.$ is $\gamma$-good) does not depend on $x$.

For the following lemma let $\ell$ be a multiple of $3, \Gamma:=\frac{\ell}{3} Z^{d}$ and $\ell^{\prime} \geq \ell$. By $\Omega_{\text {good }}$ we denote the subset of those $\omega \in \Omega$ for which no two disjoint $\gamma$-bad cubes of size $\ell$ with center in $\Gamma \cap \Lambda_{3 \ell^{\prime}}$ exist. This means that diam $\Gamma_{b} \leq \frac{5}{3} \ell$ for the union $\Gamma_{b}$ of $\gamma$-bad boxes.

LFMMA 5.2. (i) If $\mathbf{P}\left(\Lambda_{\ell} \gamma\right.$-good $) \geq 1-\eta$, then $\mathbf{P}\left(\Omega_{\text {good }}\right) \geq 1-c\left(\frac{\ell^{\prime}}{\tau}\right)^{2 d} \eta^{2}$.

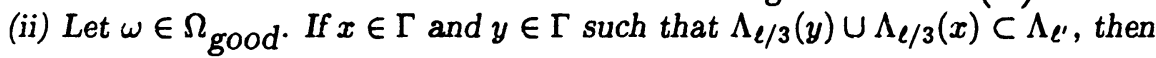

$$
\left\|\chi_{\ell / 3, x} R_{3 \ell^{\prime}}(E+\mathrm{i} \varepsilon) \chi_{\ell / 3, y}\right\| \leq\left(3^{d} \mathrm{e}^{-\gamma \ell}\right)^{3|x-y| \ell^{-1}-4}\left\|R_{3 \ell^{\prime}}(E+\mathrm{i} \varepsilon)\right\|
$$

Proof. (i) follows from elementary combinatorics.

(ii) At first, suppose that $\Lambda_{\ell}(x)$ is $\gamma$-good and $\Lambda_{\ell}(x) \cap \Lambda_{\ell / 3}(y)=\emptyset$. Then $\varphi_{\ell, x} \chi_{\ell / 3, y}=$ 0 and therefore the geometric resolvent equation implies

$$
\begin{aligned}
\chi_{\ell / 3, x} R_{3 \ell^{\prime}} \chi_{\ell / 3, y} & =\chi_{\ell / 3, x} R_{\ell, x} W\left(\varphi_{\ell, x}\right) R_{3 \ell^{\prime}} \chi_{\ell / 3, y} \\
& =\chi_{\ell / 3, x} R_{\ell, x} W\left(\varphi_{\ell, x}\right) \sum_{\tilde{x}_{j} \in \Gamma \cap\left(\Lambda_{\ell}(x) \backslash \Lambda_{\ell / 9}(x)\right)} \chi_{\ell / 3, \bar{x}_{j}} R_{3 \ell^{\prime}} \chi_{\ell / 3, y} .
\end{aligned}
$$

Let $x_{1}$ be chosen from the $\tilde{x}_{j}$ such that $\left\|\chi_{\ell / 3, \tilde{x}_{j}} R_{3 \ell^{\prime}} \chi_{\ell / 3, y}\right\|$ becomes maximal. Thus, using also that $\Lambda_{\ell}(x)$ is $\gamma$-good,

$$
\left\|\chi_{\ell / 3, x} R_{3 \ell^{\prime}} \chi_{\ell / 3, y}\right\| \leq 3^{d} \mathrm{e}^{-\gamma \ell}\left\|\chi_{\ell / 3, x_{1}} R_{3 \ell^{\prime}} \chi_{\ell / 3, y}\right\| .
$$

This process can now be repeated to construct points $x_{1}, \ldots, x_{k} \in \Gamma$ as long as $\Lambda_{\ell, x_{k-1}}$ is $\gamma$-good and does not hit $\Lambda_{\ell / 3}(y)$ or $\partial \Lambda_{3 \ell^{\prime}}$. The same type of estimate (using adjoints) can be applied to $y$ as a starting point, yielding points $y_{1}, \ldots, y_{j}$. The process moves in steps of $\ell / 3$. Therefore, it follows from $\Lambda_{\ell / 3}(y) \cup \Lambda_{\ell / 3}(x) \subset \Lambda_{\ell^{\prime}}$ and the assumption on diam $\Gamma_{b}$ that $j+k \geq 3|x-y| \ell^{-1}-4$ iterations can be performed before the process stops on both sides. Estimating finally $\left\|\chi_{\ell / 3, x_{h}} R_{3 \ell^{\prime}} \chi_{\ell / 3, y_{j}}\right\| \leq\left\|R_{3 \ell^{\prime}}\right\|$ yields (5.1). The lemma is proved.

LFMMA 5.3. Let $\xi>0$ and assume that $E$ satisfies a Wegner estimate as given in Theorem 3.1. Then there exist constants $c_{1}, c_{2}$ and $c_{3}$ such that: If

$$
\mathbf{P}\left(\Lambda_{\ell} \gamma \text {-good }\right) \geq 1-\eta
$$

for some multiple $\ell$ of $3, \gamma>0$ and $\eta>0$, then

$$
\mathbf{P}\left(\Lambda_{\ell^{\prime}} \gamma^{\prime} \text {-good }\right) \geq 1-\eta^{\prime}
$$


for every multiple $\ell^{\prime}$ of 3 with $\ell^{\prime}>4 \ell$ and

$$
\begin{aligned}
& \eta^{\prime}=c_{1}\left(\frac{\ell^{\prime}}{\ell}\right)^{2 d} \eta^{2}+\frac{1}{2}\left(\ell^{\prime}\right)^{-\xi} \\
& \gamma^{\prime}=\gamma\left(1-\frac{4 \ell}{\ell^{\prime}}\right)-\frac{c_{2}}{\ell}-c_{3} \frac{\log \ell^{\prime}}{\ell^{\prime}} .
\end{aligned}
$$

Proof. Choose $\tilde{\varphi}_{\ell} \in C_{0}^{\infty}\left(\Lambda_{\ell}\right)$ with $\tilde{\varphi}_{\ell}(y)=1$ for $\operatorname{dist}\left(y, \partial \Lambda_{\ell}\right) \geq \delta_{0}$ and such that $\left|\partial_{i} \tilde{\varphi}_{\ell}\right|$ and $\left|\partial_{i} \partial_{j} \tilde{\varphi}_{\ell}\right|$ are uniformly bounded in $\ell$ for all $i, j$. The geometric resolvent equation implies

$$
\begin{aligned}
W\left(\varphi_{\ell^{\prime}}\right) R_{3 \ell^{\prime}} \chi_{\ell^{\prime} / 3} & =W\left(\varphi_{\ell^{\prime}}\right) \tilde{\varphi}_{\ell^{\prime}} R_{3 \ell^{\prime}} \chi_{\ell^{\prime} / 3} \\
& =W\left(\varphi_{\ell^{\prime}}\right) R_{\ell^{\prime}} \chi_{\ell^{\prime} / 3}+W\left(\varphi_{\ell^{\prime}}\right) R_{\ell^{\prime}} W\left(\tilde{\varphi}_{\ell^{\prime}}\right) R_{3 \ell^{\prime}} \chi_{\ell^{\prime} / 3}
\end{aligned}
$$

Thus

$$
\begin{gathered}
\left\|W\left(\varphi_{\ell^{\prime}}\right) R_{\ell^{\prime}} \chi_{\ell^{\prime} / 3}\right\| \\
\leq\left\|W\left(\varphi_{\ell^{\prime}}\right) R_{3 \ell^{\prime}} \chi_{\ell^{\prime} / 3}\right\|+C\left(\left\|R_{\ell^{\prime}}\right\|+\left\|\nabla R_{\ell^{\prime}}\right\|\right)\left\|W\left(\tilde{\varphi}_{\ell^{\prime}}\right) R_{3 \ell^{\prime}} \chi_{\ell^{\prime} / 3}\right\| .
\end{gathered}
$$

To further estimate

$$
\left\|W\left(\varphi_{\ell^{\prime}}\right) R_{3 \ell^{\prime}} \chi_{\ell^{\prime} / 3}\right\| \leq\left\|\left(\Delta \varphi_{\ell^{\prime}}\right) R_{3 \ell^{\prime}} \chi_{\ell^{\prime} / 3}\right\|+2\left\|\left(\nabla \varphi_{\ell^{\prime}}\right) \cdot \nabla R_{3 \ell^{\prime}} \chi_{\ell^{\prime} / 3}\right\|
$$

we first note that by covering $\operatorname{supp}\left(\Delta \varphi_{\ell^{\prime}}\right)$ and $\Lambda_{\ell^{\prime} / 3}$ with cubes of sidelength $\ell / 3$ one can conclude from Lemma 5.2 that

$$
\left\|\left(\Delta \varphi_{\ell^{\prime}}\right) R_{3 \ell^{\prime}} \chi_{\ell^{\prime} / 3}\right\| \leq C\left(\frac{\ell^{\prime}}{\ell}\right)^{2 d}\left(3^{d} \mathrm{e}^{-\gamma \ell}\right)^{\frac{\ell^{\prime}-4}{\zeta}}\left\|R_{3 \ell^{\prime}}\right\|
$$

with probability $\mathbf{P} \geq 1-c\left(\ell^{\prime} / \ell\right)^{2 d} \eta^{2}$.

To control the second term on the r.h.s. of (5.4) we use the following standard estimate: There exist constants $C_{1}$ and $C_{2}$ (independent of $L$ and $\omega$ ) such that

$$
\left\|\varphi H_{L} g\right\|^{2} \geq \frac{1}{2}\left\|\varphi \partial_{j} g\right\|^{2}-C_{1}\|\varphi g\|^{2}-C_{2}\|(\nabla \varphi) g\|^{2}
$$

for every $g \in D\left(H_{L}\right)$ and $\varphi \in C_{0}^{\infty}\left(\Lambda_{L}\right)$. This estimate uses uniform (in $\omega$ and $L$ ) relative form boundedness of $V_{\text {per }}+V_{\omega}$ with respect to $H_{L}$ and can be proven by a calculation as for example in $[35$, p. $317 \mathrm{ff}]$.

Choosing $L=3 \ell^{\prime}, \varphi=\left(\partial \varphi_{\ell^{\prime}}\right)$ and $g=R_{3 \ell^{\prime}} \chi_{\ell^{\prime} / 3} f, f \in L^{2}\left(\Lambda_{3 \ell^{\prime}}\right)$ in (5.6) yields after a simple calculation that $\left\|\left(\nabla \varphi_{\ell^{\prime}}\right) \cdot \nabla R_{3 \ell^{\prime}} \chi_{\ell^{\prime} / 3}\right\|$ can be estimated by a sum of terms of the form $\left\|\phi R_{3 \ell^{\prime}} \chi_{\ell^{\prime} / 3}\right\|$, where $\phi$ is a first or second order partial derivative of $\varphi_{\ell^{\prime}}$. All these terms can be estimated as in (5.5) and therefore by (5.4) this estimate also holds for $\left\|W\left(\varphi_{\ell^{\prime}}\right) R_{3 \ell^{\prime}} \chi_{\ell^{\prime} / 3}\right\|$. In exactly the same way we estirnate $\left\|W\left(\tilde{\varphi_{\ell^{\prime}}}\right) R_{3 \ell^{\prime}} \chi_{\ell^{\prime} / 3}\right\|$ in $(5.2)$ and arrive at

$$
\left\|W\left(\varphi_{\ell^{\prime}}\right) R_{\ell^{\prime}} \chi \ell_{\ell^{\prime} / 3}\right\| \leq C\left(\frac{\ell^{\prime}}{\ell}\right)^{2 d}\left(3^{d} \mathrm{e}^{-\gamma \ell}\right)^{\frac{\ell^{\prime}}{7}-4}\left\|R_{3 \ell^{\prime}}\right\|\left(1+\left\|R_{\ell^{\prime}}\right\|+\left\|\nabla R_{\ell^{\prime}}\right\|\right)
$$

with probability $\mathbf{P} \geq 1-c_{1}\left(\frac{\ell^{\prime}}{\ell}\right)^{2 d} \eta^{2}$ 
By the Wegner estimate (3.1) we know with probability $\mathbf{P} \geq 1-2 C_{W} \varepsilon L^{2 d}$ that $\operatorname{dist}\left(E, \sigma\left(H_{L}\right)\right) \geq \varepsilon$, i.e. $\left\|R_{L}\right\| \leq 1 / \varepsilon$. The choice $L=3 \ell^{\prime}$ and $\varepsilon=\frac{1}{8 C_{W}} 3^{-2 d}\left(\ell^{\prime}\right)^{-\xi-2 d}$ gives

$$
\left\|R_{3 \ell^{\prime}}\right\| \leq C\left(\ell^{\prime}\right)^{\xi+2 d} \text { with } \mathbf{P} \geq 1-\frac{1}{4}\left(\ell^{\prime}\right)^{-\xi}
$$

Furthermore

$$
\nabla R_{L}(E+\mathrm{i} \varepsilon)=\nabla R_{L}(c)+(E+\mathrm{i} \eta-c) \nabla R_{L}(c) R_{L}(E+\mathrm{i} \varepsilon)
$$

where arguments using relative form boundedness guarantee the existence of $c \in \mathrm{R}$. with $c+1 \leq \inf \sigma\left(H_{L}\right)$ and $\left\|\nabla R_{L}(c)\right\| \leq C$ uniformly in $L$ and $\omega$. Thus

$$
\left\|R_{L}\right\|+\left\|\nabla R_{L}\right\| \leq C\left(1+\frac{1}{\varepsilon}\right) \quad \text { with } \quad \mathbf{P} \geq 1-2 C_{W} \varepsilon L^{2 d} .
$$

The choice $L=\ell^{\prime}$ and $\varepsilon=\frac{1}{8 C_{W}}\left(\ell^{\prime}\right)^{-\xi-2 d}$ proves that

$$
\left\|R_{\ell^{\prime}}\right\|+\left\|\nabla R_{\ell^{\prime}}\right\| \leq C\left(\ell^{\prime}\right)^{\xi+2 d} \text { with } \quad \mathbf{P} \geq 1-\frac{1}{4}\left(\ell^{\prime}\right)^{-\xi} .
$$

From (5.7), (5.8) and (5.9) we get

$$
\left\|W\left(\varphi_{\ell^{\prime}}\right) R_{\ell^{\prime}} \chi_{\ell^{\prime} / 3}\right\| \leq C\left(\frac{\ell^{\prime}}{\ell}\right)^{2 d}\left(3^{d} \mathrm{e}^{-\gamma \ell}\right)^{\frac{\ell^{\prime}}{\ell}-4}\left(\ell^{\prime}\right)^{2 \xi+4 d}
$$

with $\mathbf{P} \geq 1-c_{1}\left(\frac{\ell^{\prime}}{\ell}\right)^{2 d} \eta^{2}-\frac{1}{2}\left(\ell^{\prime}\right)^{-\xi}$. A simple calculation shows that the r.h.s. of $(5.10)$ is

$$
\leq \exp \left(-\ell^{\prime}\left[\gamma\left(1-\frac{4 \ell}{\ell^{\prime}}\right)-\frac{d \log 3}{\ell}-\frac{\log C}{\ell^{\prime}}-\frac{(2 \xi+6 d) \log \ell^{\prime}}{\ell^{\prime}}\right]\right) .
$$

The proof of Lemma 5.3 is therefore completed by choosing $c_{3}=2 \xi+6 d$ and estimating $\frac{d \log 3}{\ell}+\frac{\log C}{\ell^{\prime}} \leq \frac{c_{2}}{\ell}$.

LFMMA 5.4. Let $a \in \partial \Sigma$ and $0<\xi<2 \tau-d$ under the assumptions of Theorem 1.1 or let $a=\inf \Sigma$ and $\xi>0$ under the assumptions of Theorem 1.2. Let $\alpha \in\left(1, \frac{2 d+2 \xi}{2 d+\xi}\right)$.

Then there exists a neighborhood $I$ of a such that for every $E \in I$ there is an $\ell^{*} \in \mathbf{N}$ and $\gamma>0$ with the property

$$
\mathbf{P}\left(\Lambda_{\ell_{k}} \text { is } \gamma \text {-good }\right) \geq 1-\ell_{k}^{-\xi} \text { for } k=0,1,2, \ldots
$$

where $\ell_{0} \geq \ell^{*}$ is an arbitrary multiple of 3 and $\ell_{k}=\left[\ell_{0}^{\alpha^{k}}\right]_{3}$. Here $[x]_{3}$ denotes the largest multiple of 3 which is smaller or equal to $x$.

The rest of this section will be used to prove Lemma 5.4. Under the given assumptions we know from Propositions 4.1 resp. 4.2 that there exist $\beta>0$ and $\ell^{*}$ such that

$$
\operatorname{dist}\left(a, \sigma\left(H_{\ell}\right)\right) \geq \ell^{\beta-2} \quad \text { with } \quad \mathbf{P} \geq 1-\ell^{-\xi}
$$

for $\ell \geq \ell^{*}$, i.e. for $E \in I:=\left(a-\frac{1}{2} \ell^{\beta-2}, a+\frac{1}{2} \ell^{\beta-2}\right)$ we have

$$
\operatorname{dist}\left(E, \sigma\left(H_{\ell}\right)\right) \geq \frac{1}{2} \ell^{\beta-2} \quad \text { with } \quad \mathbf{P} \geq 1-\ell^{-\xi} \text {. }
$$


We will now use the following Lemma whose proof is given in an Appendix and based on an improved version of the Combes-Thomas method as introduced in [1].

LFMMA 5.5. Let $I, \xi$ and $\beta$ be given as above. Then for $E \in I$ it holds with probability $\mathbf{P} \geq 1-\ell^{-\xi}$ that

$$
\sup _{\varepsilon \neq 0}\left\|W\left(\varphi_{\ell}\right) R_{\ell}(E+\mathrm{i} \varepsilon) \chi \ell / 3\right\| \leq \mathrm{e}^{-\ell^{\dot{\beta}}}
$$

if $0<\tilde{\beta}<\beta / 2$ and $\ell \geq \ell^{*}$ with $\ell^{*}$ sufficiently large.

Therefore, if we choose $\ell_{0} \geq \ell^{*}$ a multiple of $3, \gamma_{0}=\ell_{0}^{\bar{\beta}-1}$, and $\eta_{0}=\ell_{0}^{-\xi}$, then

$$
\mathbf{P}\left(\Lambda_{\ell_{0}} \text { is } \gamma_{0} \text {-good }\right) \geq 1-\ell_{0}^{-\xi} \text {. }
$$

With this $\ell_{0}$ and $\ell_{k}=\left[\ell_{0}^{\alpha^{k}}\right]_{3}$ we have $\ell_{k+1}>4 \ell_{k}$ for all $k$ if $\ell^{*}$ is large enough. Since $E$ satisfies a Wegner estimate by Theorem 3.1 we can therefore inductively apply Lemma 5.3 and conclude that

$$
\mathbf{P}\left(\Lambda_{\ell_{k}} \text { is } \gamma_{k} \text {-good }\right) \geq 1-\eta_{k},
$$

where

$$
\gamma_{k+1}=\gamma_{k}\left(1-\frac{4 \ell_{k}}{\ell_{k+1}}\right)-\frac{c_{2}}{\ell_{k}}-c_{3} \frac{\log \ell_{k+1}}{\ell_{k+1}}
$$

and

$$
\eta_{k+1}=c_{1}\left(\frac{\ell_{k+1}}{\ell_{k}}\right)^{2 d} \ell_{k}^{-2 \xi}+\frac{1}{2} \ell_{k+1}^{-\xi} .
$$

By a calculation as in [2, Proof of Lemma A.3] one can check that the $\gamma_{k}$ are strictly bounded from below by a positive number, i.e.

$$
\gamma_{k} \geq \gamma>0 \text { for all } k \text {. }
$$

From the definition of $\ell_{k}$ we get $\ell_{k+1} \leq\left(2 \ell_{k}\right)^{\alpha}$ if $\ell^{*}$ is large enough. This and (5.14) yield the estimate

$$
\eta_{k+1} \leq\left(C \ell_{k+1}^{2 d+\xi-(2 d+2 \xi) / \alpha}+\frac{1}{2}\right) \ell_{k+1}^{-\xi}
$$

The term in brackets is bounded by 1 for all $k$ if $\ell^{*}$ is sufficiently large since $\alpha \in$ $(1,(2 d+2 \xi) /(2 d+\xi))$, i.e.

$$
\eta_{k+1} \leq \ell_{k+1}^{-\xi} \quad \text { for } \quad k=0,1,2, \ldots
$$

Lemma 5.4 follows from (5.12), (5.15) and (5.16).

\section{FROM FINITE BOXES TO THE WHOLE SPACE}

The goal of this section is to turn the finite box resolvent estimates proven in Section 5 into the basic estimates for $R(z, \omega)=(H(\omega)-z)^{-1}$ needed to prove localization and exponential decay for eigenfunctions. The main result is

THFORFM 6.1. Let $Q: \mathrm{R}^{d} \rightarrow \mathrm{R}$. be bounded, measurable and of compact support. Under the assumptions of Theorem 1.1 there exists a neighborhood $U$ of $\partial \Sigma$ and under 
the assumptions of Theorem 1.2 there exists a neighborhood $U$ of inf $\Sigma$ such that the following holds with probability one:

(a) For almost every $E \in U$

$$
\sup _{\varepsilon \neq 0}\|R(E+\mathrm{i} \varepsilon) Q\|<\infty
$$

(b) For almost every $E \in U$ there exist $C$ and $\gamma>0$ such that

$$
\sup _{\varepsilon \neq 0}\left\|\chi_{x} R(E+\mathrm{i} \varepsilon) Q\right\| \leq C \mathrm{e}^{-\gamma|x|}
$$

for every $x \in \mathrm{Z}^{d}$. Here $\chi_{x}:=\chi_{\Lambda_{1}(x)}$.

Although the above statements could be proven "from first principles" (compare [2, Theorems 2.1 and 2.4], we have decided to base our proof on the following deterministic fact:

Proposition 6.2. Let $H=-\Delta+V$ where $V_{-}$is in the Kato class and $V_{+}$is locally in the Kato class. Then there exists a subset $S_{0} \subset \mathrm{R}$ of full measure, i.e. $\left|\mathrm{R} \backslash S_{0}\right|=0$, such that

$$
\sup _{\varepsilon \neq 0}\left\|\chi_{A}(H-E-\mathrm{i} \varepsilon)^{-1} \chi_{B}\right\|<\infty
$$

for every $E \in S_{0}$ and every pair of compact subsets $A$ and $B$ of $\mathrm{R}^{d}$.

Results of this type go back to de Brange's work [5]. The above formulation is found in [33, Lemma 3]. The Kato class includes all the potentials discussed in the present paper.

As additional preparation for the proof of Theorem 6.1 we need

LFMMA 6.3. Let $I$ be as in Lemma 5.4 and $E \in I$ be fixed. With $\ell^{*}, \ell_{k}$ and $\gamma>0$ as in Lemma 5.4 let $\psi_{k}:=\chi_{3 \ell_{k}}-\chi_{3 \ell_{k-1}}$. Then, if $\ell^{*}$ is sufficiently large, with probability $\mathbf{P} \geq 1-\ell_{k}^{-\xi}$ it holds that

$$
\sup _{\varepsilon \neq 0}\left\|\psi_{k} R_{g \ell_{k}}(E+\mathrm{i} \varepsilon) W\left(\varphi g \ell_{k}\right)\right\| \leq \mathrm{e}^{-\frac{g}{2} \gamma \ell_{h}}
$$

and

$$
\sup _{\varepsilon \neq 0}\left\|\psi_{k} R_{9 \ell_{k}}(E+\mathrm{i} \varepsilon) Q\right\| \leq C\|Q\|_{\infty} \ell_{k}^{3 d-d / \alpha+\xi} \mathrm{e}^{-5 \gamma \ell_{k-1}}
$$

Proof. By Lemma 5.4 we have $\mathbf{P}\left(\Lambda_{\ell_{h-1}} \gamma\right.$-good $) \geq 1-\ell_{k-1}^{-\xi}$, and therefore we can apply Lemma 5.3 with $\ell=\ell_{k-1}$ and $\ell^{\prime}=9 \ell_{k}$, yielding

$$
\left\|\psi_{k} R_{9 \ell_{k}} W\left(\varphi_{9 \ell_{k}}\right)\right\| \leq \mathrm{e}^{-9 \gamma^{\prime} \ell_{k}}
$$

with

$$
\mathbf{P} \geq 1-c_{1}\left(\frac{9 \ell_{k}}{\ell_{k-1}}\right)^{2 d} \ell_{k-1}^{-2 \xi}-\frac{1}{2}\left(9 \ell_{k}\right)^{-\xi}
$$

where

$$
\gamma^{\prime}=\gamma\left(1-\frac{4 \ell_{k-1}}{9 \ell_{k}}\right)-\frac{c_{2}}{\ell_{k-1}}-c_{3} \frac{\log 9 \ell_{k}}{9 \ell_{k}}
$$


If $\ell^{*}$ is sufficiently large, then $\gamma^{\prime} \geq \gamma / 2$ for all $k$ and, by a calculation as in the proof of Lemma 5.4, the probability in (6.3) can be estimated by $\mathbf{P} \geq 1-\ell_{k}^{-\xi}$. This proves (6.1).

To prove (6.2) we use Lemma 5.2 with $\ell=\ell_{k-1}, \ell^{\prime}=3 \ell_{k}$ and $\eta=\ell_{k-1}^{-\xi}$ (possible by Lemma 5.4). We have $|Q| \leq\|Q\|_{\infty} \chi \ell_{\ell_{k-1} / 3,0}$ if $\ell^{*}$ is sufficiently large, and $\operatorname{supp} \psi_{k}$ can be covered with $C\left(\ell_{k} / \ell_{k-1}\right)^{d} \leq C \ell_{k}^{d-d / \alpha}$ cubes of sidelength $\ell_{k-1} / 3$, all of whose centers having at least distance $3 \ell_{k-1}$ to 0 . Thus (5.1) proves

$$
\left\|\psi_{k} R_{9 \ell_{k}} Q\right\| \leq C\|Q\|_{\infty} \ell_{k}^{d-d / \alpha}\left(3^{d} \mathrm{e}^{-\gamma \ell_{k-1}}\right)^{5}\left\|R_{9 \ell_{b}}\right\|
$$

with $\mathbf{P} \geq 1-c\left(\ell_{k} / \ell_{k-1}\right)^{2 d} \ell_{k-1}^{-2 \xi}$. Wegner's estimate yields

$$
\left\|R_{g \ell_{k}}\right\| \leq C \ell_{k}^{\xi+2 d} \text { with } \quad \mathbf{P} \geq 1-\frac{1}{2} \ell_{k}^{-\xi} .
$$

This and (6.4) imply (6.2) using $C\left(\ell_{k} / \ell_{k-1}\right)^{2 d} \ell_{k-1}^{-2 \xi}+\frac{1}{2} \ell_{k}^{-\xi} \leq \ell_{k}^{-\xi}$ for large $\ell^{*}$.

We are now in a position to complete the Proof of Theorem 6.1(a): Take $U$ to be the (union of) neighborhood(s) provided by Lemma 5.4. Since $\sum_{k} \ell_{k}^{-\xi}<\infty$ we conclude from the Borel Cantelli lemma that for every fixed $E \in U$ and almost every $\omega$ there exists $k_{0}=k_{0}(\omega)$ such that (6.1) and (6.2) hold for every $k \geq k_{0}$. For these $\omega$ and arbitrary $f \in L^{2}\left(\mathbf{R}^{d}\right)$ we get the following estimate using the geometric resolvent equation and Lemma 6.3:

$$
\begin{aligned}
& \left\|\left(1-\chi_{3 \ell_{h_{0}}}\right) R .(E+\mathrm{i} \varepsilon) Q f\right\|^{2} \\
& =\sum_{k \geq k_{0}}\left\|\psi_{k} R .(E+\mathrm{i} \varepsilon) Q f\right\|^{2} \\
& \leq 2 \sum_{k \geq k_{0}}\left(\left\|\psi_{k} R_{9 \ell_{k}}(E+\mathrm{i} \varepsilon) Q f\right\|^{2}+\left\|\psi_{k} R_{9 \ell_{k}}(E+\mathrm{i} \varepsilon) W\left(\varphi_{9 \ell_{k}}\right)\right\|^{2}\left\|\psi_{k+1} R(E+\mathrm{i} \varepsilon) Q f\right\|^{2}\right) \\
& \leq C\|Q\|_{\infty}^{2} \sum_{k \geq k_{0}}\left(\ell_{k}^{3 d-d / \alpha+\xi} \mathrm{e}^{-5 \gamma \ell_{k-1}}\right)^{2}\|f\|^{2}+2 \mathrm{e}^{-9 \gamma \ell_{k_{0}}} \sum_{k \geq k_{0}+1}\left\|\psi_{k} R(E+\mathrm{i} \varepsilon) Q f\right\|^{2} .
\end{aligned}
$$

If we choose $k_{0}$ sufficiently large such that $2 \mathrm{e}^{-9 \gamma \ell_{m_{0}}} \leq \frac{1}{2}$, then it follows that

$$
\begin{aligned}
\left\|\left(1-\chi_{3 k_{0}}\right) R(E+\mathrm{i} \varepsilon) Q f\right\|^{2} & \leq 2 C\|Q\|_{\infty}^{2} \sum_{k \geq k_{0}}\left(\ell_{k}^{3 d-d / \alpha+\xi^{-5 \gamma \ell_{k-1}}}\right)^{2}\|f\|^{2} \\
& \leq C\|f\|^{2}
\end{aligned}
$$

uniformly in $\varepsilon \neq 0$. Thus we have proven that if $E \in U$ is fixed, then for almost every $\omega$ there exists a compact subset $K=K(\omega) \subset \mathbf{R}^{d}$ such that

$$
\sup _{\varepsilon \neq 0}\left\|\left(1-\chi_{\kappa}\right) R(E+\mathrm{i} \varepsilon, \omega) Q\right\|<\infty
$$

Fubini's theorem implies that the following holds with probability 1: For almost every $E \in U$ there exists a compact subset $K=K(E) \subset \mathrm{R}^{d}$ such that (6.6) holds. For almost all of these $E$ 's it is by Proposition 6.2 also true that

$$
\sup _{\varepsilon \neq 0}\left\|\chi_{K(E)} R(E+\mathrm{i} \varepsilon, \omega) Q\right\|<\infty
$$


This combines to prove part (a) of Theorem 6.1, i.e. the existence of a subset $U_{0} \subset U$ with $\left|U \backslash U_{0}\right|=0$ and such that $\sup _{\varepsilon \neq 0}\|R .(E+\mathrm{i} \varepsilon) Q\|<\infty$ for $E \in U_{0}$ with probability 1.

For the following proof of part (b) of Theorem 6.1 assume that $E \in U_{0}$. Given $x \in \mathrm{Z}^{d}$, choose $k$ such that $x \in \Lambda_{3 \ell_{k}} \backslash \Lambda_{3 \ell_{k-1}}$ and assume that supp $Q \subset \Lambda_{\ell_{k-1} / 3}$. One more application of the geometric resolvent equation gives

$$
\left\|\chi_{x} R Q\right\| \leq\left\|\chi_{x} R_{9 \ell_{k}} Q\right\|+\left\|\chi_{x} R_{9 \ell_{k}} W\left(\varphi_{9 \ell_{k}}\right) R Q\right\| .
$$

From (a) above and (6.1) we se that the second term on the r.h.s. can be estimated by

$$
\left\|\chi_{x} R_{9 \ell_{k}} W\left(\varphi_{9 \ell_{k}}\right) R Q\right\| \leq C\left\|\chi_{x} R_{9 \ell_{k}} W\left(\varphi_{9 \ell_{k}}\right)\right\| \leq C \mathrm{e}^{-\frac{8}{2} \gamma \ell_{k}} \leq C \mathrm{e}^{-\frac{8}{2} \gamma|x|}
$$

with $\mathbf{P} \geq 1-\ell_{k}^{-\xi}$. Lemma 5.2, Wegner's estimate (compare (6.5), and some calculations are used to estimate the first term on the r.h.s. of (6.7):

$$
\begin{aligned}
\left\|\chi_{x} R_{9 \ell_{h}} Q\right\| & \leq\|Q\|_{\infty}\left(3^{d}\right)^{9 \ell_{k} / \ell_{k-1}} \mathrm{e}^{-\frac{1}{\gamma} \gamma|x|}\left\|R_{9 \ell_{k}}\right\| \\
& \leq C\|Q\|_{\infty} \ell_{k}^{\xi+2 d} \mathrm{e}^{-\frac{1}{3} \gamma|x|+9 d(\log 3) \ell_{h} / \ell_{k-1}} \\
& \leq C\|Q\|_{\infty}|x|^{\alpha(\xi+2 d)} \mathrm{e}^{-\frac{1}{c} \gamma|x|+C|x|^{\alpha-1}} \\
& \leq \mathrm{e}^{-\gamma^{\prime}|x|}
\end{aligned}
$$

for $0<\gamma^{\prime}<\gamma / 3$ and $|x|$ sufficiently large (note $\left.\alpha<2\right)$ with probability $\mathbf{P} \geq 1-$ $C\left(3 \ell_{k} / \ell_{k-1}\right)^{2 d} \ell_{k-1}^{-2 \xi}-\frac{1}{2} \ell_{k}^{-\xi} \geq 1-C \ell_{k}^{-\xi}$ (compare (5.16). Inserting (6.8) and (6.9) in (6.7) we see that

$$
\left\|\chi_{x} R Q\right\| \leq C \mathrm{e}^{-\gamma|x|}
$$

holds for every $E \in U_{0}$ and large $|x|$ with $\mathbf{P} \geq 1-C \ell_{k}^{-\xi}$. Summability of $\ell_{k}^{-\xi}$ implies that for every $E \in U_{0}$ with probability 1 there exists $k_{0}$ such that (6.10) holds for every $x \in \mathrm{R}^{d} \backslash \Lambda_{3 \ell_{n_{0}}}$.

Fubini's Theorem shows that with probability $1(6.10)$ holds for large $|x|$ and almost every $E \in U_{0}$, and therefore almost every $E \in U$. Finally, part (a) of Theorem 6.1 allows us to drop the restriction 'large $|x|$ ' from the preceding statement, thus completing the proof of part (b).

\section{GENERALIZED EIGENFUNCTIONS AND SPECTRAL AVERAGING}

In this section we will introduce two more basic results from spectral theory which will be used in the conclusion of the proofs of our main results.

Theorem 6.1 says that certain properties of the resolvent hold with probability one for almost every $E$ in a given set. To use this in the proof of our main results we will need to know that the exceptional energies, where these properties do not hold, can be ignored, i.e. with probability one have no effect on the spectrum of $H(\omega)$. To this end we will use a result on spectral averaging, which is essentially due to [21] with generalized versions given in [2] and [3].

Proposition 7.1. Let $H_{0}=-\Delta+W$, where $W$ is locally uniformly in $L_{l o c}^{p}\left(\mathrm{R}^{d}\right)$, where $p=2$ if $d \leq 3$ and $p>\frac{d}{2}$ if $d \geq 4$. Let $f$ be defined as in Section $1, H_{\lambda}:=H_{0}+\lambda f$ and $E_{\lambda}(\cdot)$ be the spectral resolution of $H_{\lambda}$.

Then for any Borel set $L$ with $|L|=0$ it holds that $E_{\lambda}(L)=0$ for almost every $\lambda$. 
Since $f$ is relatively bounded with respect to $-\Delta$ and $g \geq \chi:=\chi_{\Lambda_{0}(0)}$ it is seen that $H_{\lambda}$ satisfies the general assumptions used in the proof of spectral averaging in [3]. By Proposition A.2.2 of [2] it also holds that $\left\{g\left(H_{\lambda}\right) \chi \phi: g \in L^{\infty}(\mathrm{R}), \phi \in L^{2}\left(\mathrm{R}^{d}\right)\right\}$ is dense in $L^{2}\left(\mathrm{R}^{d}\right)$. Thus Proposition 7.1 is a special case of Corollary 1.3 in [3].

We will also use a result on generalized eigenfunctions of Schrödinger operators. First, recall that every self-adjoint operator $H$ in a separable Hilbert space $\mathcal{H}$ has an ordered spectral representation, i.e. a unitary operator

$$
U: \mathcal{H} \longrightarrow \bigoplus_{j=1}^{N} L^{2}\left(\mathrm{R}, d \rho_{j}\right) \quad(N \in \mathrm{N} \cup\{\infty\})
$$

such that $U H U^{-1}=M_{i d}$, the operator of multiplication by the variable in $\oplus_{j} L^{2}\left(\mathrm{R}, d \rho_{j}\right)$. Here the $\rho_{j}$ are bounded Borel measures and $\rho_{j+1}$ is absolutely continuous with respect to $\rho_{j}$ for all $j$ (see e.g. [36, Theorem 8.1]). The measure $\mu:=\rho_{1}$ is a spectral measure for $H$, i.e. for Borel sets $L$ we have $\mu(L)=0$ if and only if $E(L)=0$, where $E(\cdot)$ is the spectral resolution for $H$ [25].

For the following proposition let $H_{s}^{1}=\left\{f \mid\langle x\rangle^{8} f \in H^{1}\right\}$, where $H^{1}$ is the first order $\left(L^{2}-\right)$ Sobolev space over $R^{d}$ and $\langle x\rangle=\left(1+|x|^{2}\right)^{1 / 2}$.

PROPOSITION 7.2. Let $H=-\Delta+V$ in $L^{2}\left(\mathbf{R}^{d}\right)$, where $V$ is uniformly locally in $L^{p}$ with $p=2$ if $d \leq 3$ and $p>\frac{d}{2}$ if $d \geq 4$. Let $\mu$ be a spectral measure and $s>d / 2$.

Then for $\mu$-almost every $E \in R$ there exists a non-trivial weak solution $\varphi$ of $H \varphi=E \varphi$ with $\varphi \in H_{-8}^{1}$.

Results of this type are quite standard and follow from the theory of expansions in generalized eigenfunctions. In the concrete case we may use that $V$ is $-\Delta$-bounded with relative bound 0 . Theorem 3.6 of [24] shows that under this assumption $\langle p\rangle\langle x\rangle^{-s}(H-$ $z)^{-m}$ is a Hilbert-Schmidt operator for $s>d / 2, z \in \rho(H)$, and $m$ sufficiently large (depending on $d$ ), here $\langle p\rangle=F^{-1}\langle x\rangle F, F$ the Fourier transform.

This allows to apply the results of [25], which, in particular, establish the existence of weak solutions $\varphi$ of $H \varphi=E \varphi$ with $\varphi \in\left\{\langle x\rangle^{\circ}\langle p\rangle^{-1} f \mid f \in L^{2}\right\}=H_{-8}^{1}$ for $\mu$-almost every $E$. Non-triviality of the $\varphi$ follows from the expansion theorem in [25].

\section{PROOF OF LACALIZATION}

Let $U$ be the neighborhood of $\partial \Sigma$ resp. inf $\Sigma$ provided in Theorem 6.1. To $\omega \in \Omega$ let

$$
\begin{aligned}
\tilde{S_{\omega}} & :=\left\{E \in U: \text { for all } y \in \mathrm{Z}^{d} \text { there exist } C, \gamma>0\right. \text { such that } \\
& \left.\sup _{\varepsilon \neq 0}\left\|\chi_{x} R(E+\mathrm{i} \varepsilon, \omega) \chi_{y}\right\| \leq C \mathrm{e}^{-\gamma|x|} \text { for all } x \in \mathrm{Z}^{d}\right\}
\end{aligned}
$$

and

$$
S_{\omega}:=\tilde{S_{\omega}} \backslash\{\text { eigenvalues of } H(\omega)\}
$$

For energies in $S_{i}$ we will be able to establish exponential decay of generalized eigenfunctions using

LFMMA 8.1. Let $E \in S_{\omega}$ and $\varphi \in H_{-8}^{1}$ be a weak solution of $(H(\omega)+\lambda f) \varphi=E \varphi$. Then there exist $C$ and $\gamma>0$ such that

$$
|\varphi(y)| \leq C \mathrm{e}^{-\gamma|y|} \quad \text { for every } y \in \mathrm{R}^{d} .
$$


Proof. For $R>0$ choose functions $g_{R} \in C_{0}^{\infty}(|x|<R)$ with $g_{R}(x)=1$ for $|x| \leq R-1$ and such that $\left|\nabla g_{R}\right| \leq C$ and $\left|\Delta g_{R}\right| \leq C$ uniformly in $R$.

By the spectral theorem and dominated convergence it holds for general self-adjoint operators $H$ and their spectral resolution $P$ that $s-\lim _{e \rightarrow 0}(H-E-\mathrm{i} \varepsilon)^{-1}(H-E)=$ $I-P(\{E\})$. Since $E$ is not an eigenvalue of $H(\omega)$ it follows that

$$
\lim _{\varepsilon \rightarrow 0} R(E+\mathrm{i} \varepsilon, \omega)(H(\omega)-E) g_{R} \varphi=g_{R} \varphi
$$

Since $(H(\omega)-E) \varphi=-\lambda f \varphi$ we have $(H(\omega)-E) g_{R} \varphi=W\left(g_{R}\right) \varphi+g_{R}(H(\omega)-E) \varphi=$ $W\left(g_{R}\right) \varphi-\lambda f \varphi$ for large $R$. Thus we can use (8.1) to get that for arbitrary $y \in \mathrm{Z}^{d}$ and $R$ large

$$
\begin{aligned}
\left\|\chi_{y} \varphi\right\| & =\left\|\chi_{y} g_{R} \varphi\right\| \\
& \leq \sup _{\epsilon \neq 0}\left\|\chi_{y} R(E+\mathrm{i} \varepsilon, \omega) W\left(g_{R}\right) \varphi\right\|+|\lambda| \sup _{\epsilon \neq 0}\left\|\chi_{y} R(E+\mathrm{i} \varepsilon, \omega) f \varphi\right\| .
\end{aligned}
$$

The first term on the r.h.s. of (8.2) is estimated by

$$
\left\|\left(\Delta g_{R}\right) R(E-\mathrm{i} \varepsilon, \omega) \chi_{y}\right\|\left\|\left.\varphi\right|_{|x|<R}\right\|+2\left\|\left(\nabla g_{R}\right) R(E-\mathrm{i} \varepsilon, \omega) \chi_{y}\right\|\left\|\left.(\nabla \varphi)\right|_{|x|<R}\right\| .
$$

Using $E \in S_{\omega}$ and $\varphi \in H_{-8}^{1}$ we can further estimate this by $p(R) \mathrm{e}^{-\gamma R}$ with $\gamma>0$ and a polynomial $p$. Thus, after taking the limit $R \rightarrow \infty$, we conclude from (8.2) that

$$
\left\|\chi_{y} \varphi\right\| \leq|\lambda| \sup _{\epsilon \neq 0}\left\|\chi_{y} R(E+\mathrm{i} \varepsilon, \omega) \chi_{\operatorname{supp} f}\right\|\|f \varphi\| \leq C \mathrm{e}^{-\gamma|y|}\|f \varphi\|
$$

for some $\gamma>0$, where $E \in S_{\omega}$ was used again.

For the weak solution $\varphi$ of $(H(\omega)+\lambda f) \varphi=E \varphi$ we have the subsolution estimate (e.g. $[4$, p.18])

$$
|\varphi(x)|^{2} \leq C\left\|\chi_{x} \varphi\right\|^{2}
$$

for every $x \in \mathbf{R}^{d}$. This implies $\varphi \in L_{l o c}^{\infty}$, i.e. $\|f \varphi\|<\infty$, and also $|\varphi(y)| \leq C\left\|\chi_{y} \varphi\right\| \leq$ $C \mathrm{e}^{-\gamma|y|}$ by (8.3).

From the definition of $\tilde{S_{\omega}}$ and $S_{\omega}$ and Theorem 6.1 together with a countability argument it follows that $\left|U \backslash S_{\omega}\right|=0$ with probability one, i.e. there exists $\Omega_{0} \subset \Omega$ with $\mathbf{P}\left(\Omega_{0}\right)=1$ and

$$
\left|U \backslash S_{\omega}\right|=0 \text { for every } \omega \in \Omega_{0} \text {. }
$$

Let $\omega \in \Omega_{0}$ be fixed, $H_{\lambda}:=H(\omega)+\lambda f$ and $P_{\lambda}(\cdot)$ the spectral resolution of $H_{\lambda}$. By Theorem 7.1 there exists a subset $M_{0} \subset \mathrm{R}$ with $\left|\mathrm{R} \backslash M_{0}\right|=0$ and

$$
P_{\lambda}\left(U \backslash S_{\omega}\right)=0 \text { for every } \lambda \in M_{0}
$$

Let now also $\lambda \in M_{0}$ be fixed and $\mu$ be a spectral measure for $H_{\lambda}$. By (8.5) we have

$$
\mu\left(U \backslash S_{\omega}\right)=0
$$

Proposition 7.2 yields that for $\mu$-almost every $E$ there exists a non-trivial weak solution $\varphi \in H_{-}^{1}$ of $H_{\lambda} \varphi=E \varphi$. By (8.6) and Lemma 8.1 it follows that for $\mu$-almost every $E \in U$ there exists a non-trivial exponentially decaying solution $\varphi$ of $H_{\lambda} \varphi=E \varphi$, in particular, $\varphi$ is an eigenfunction of $H_{\lambda}$. Thus $\mu$-almost every $E \in U$ is an eigenvalue of 
$H_{\lambda}$. The set of eigenvalues is countable and therefore $\left.\mu\right|_{U}$ is a point measure, i.e. $H_{\lambda}$ has pure point spectrum in $U$. All its eigenfunctions decay exponentially.

Using $\mathbf{P}\left(\Omega_{0}\right)=1$ in an application of Fubini's theorem to $\left(\omega_{0}, \omega^{\prime}\right)$ with $\omega^{\prime}=\left(\omega_{k} ; k \neq\right.$ $0)$, together with the fact that $\left|\mathbf{R} \backslash M_{0}\right|=0$ and the distribution of $\omega_{0}$ is absolutely continuous, we conclude that $H(\omega)$ has pure point spectrum in $U$ with exponentially decaying eigenfunctions for almost every $\omega$.

\section{DISCUSSION}

Our main results allow several extensions and generalizations which we have not included in the main body of the text for the sake of clarity of the presentation:

\section{Extensions. (i) Compactly supported $f$ :}

In A.2 we can allow $f$ of arbitrary compact support. This leads to minor changes in the proof of formula (1.1). Proposition 4.2 can first be applied to $\tilde{V}_{p e r}=V_{p e r}+q_{-} \sum_{k} f(\cdot-$ $k), \tilde{q}_{-}=0$, and $\tilde{f}:=\chi_{\Lambda_{1}(0)} f$. It then follows from monotonicity that (4.4) holds under the more general assumptions on $f$. Finally the definition of the set $\Omega_{\text {good }}$ in Lemma 5.2 has to be changed. It should now be the set of those $\omega \in \Omega$ such that no two $\gamma$-bad cubes of sidelength $\ell$ with centers in $\Gamma \cap \Lambda_{\ell^{\prime}}$ and of distance larger than the diameter of the support of $f$ should exist, thus guaranteeing the independence of the corresponding box hamiltonians.

The basic methods used in this paper to prove localization also apply to non-compactly supported $f$ which decay at infinity like $|x|^{-m}$ for sufficiently large $m$. This will be discussed in a separate publication [8].

\section{(ii) General lattices:}

Our results extend to the case of a $\Gamma$-periodic potential $V_{\text {per }}$ and $V_{\omega}(x)=\sum_{k \in \Gamma} q_{k}(\omega) f(\cdot-k)$ where $\Gamma$ is a general $d$-dimensional lattice, i.e.

$\Gamma=\left\{\sum_{i=1}^{d} a_{i} v_{i} ; a_{i} \in \mathrm{Z}\right\}$ for some basis $\left\{v_{1}, \ldots, v_{d}\right\}$ of $\mathbf{R}^{d}$. In Theorem 1.2 one then has to assume reflection symmetry with respect to $\Gamma$, cf. [17]. All necessary modifications in the proofs are straightforward.

\section{(iii) Closing gaps:}

Theorem 1.1 also applies to the case of a "closing gap", i.e. the situation where an upper band edge $a$ of $H_{p e r}+q_{+} \sum_{k} f(\cdot-k)$ coincides with a lower band edge of $H_{p e r}+$ $q_{-} \sum_{k} f(\cdot-k)$. For example this happens if $H_{p e r}$ has a gap of length $\alpha, f=\chi_{\Lambda_{1}(0)}$ and $q_{+}-q_{-}=\alpha$. In this case $a$ is an interior point of $\Sigma$, but nevertheless our methods show that $H_{\omega}$ is pure point $\mathbf{P}$-a.s. with exponentially decaying eigenfunctions in a neighborhood of $a$. In fact, with the method of proof of Proposition 3.2 we see that adding an auxiliary potential we get an operator whose spectrum does not contain $a$. Hence we have a Wegner estimate near $a$. For the initial length scale, we have to consider the event $\Omega_{\ell, h}=\left\{\omega \in \Omega_{;} q_{k}(\omega) \in\left[q_{-}+h, q_{+}-h\right]\right.$ for all $\left.k \in \Lambda_{\ell}(0)\right\}$ and then use the same arguments as before.

(iv) The support of $g$ is not an interval:

In fact this case is closely related to the preceding remark: we still have

$$
\bigcup_{q \in \operatorname{supp} g} \sigma\left(H_{p e r}+q \cdot \sum_{k} f(\cdot-k)\right) \subset \Sigma \subset \bigcup_{q \in\left[q_{-}, q_{+}\right]} \sigma\left(H_{p e r}+q \cdot \sum_{k} f(\cdot-k)\right)
$$


for the almost sure spectrum $\Sigma$. We can not exclude however, that boundary points of $\Sigma$ are no fluctuation boundaries. The latter are given by

$$
\partial_{\text {fluct }} \Sigma=\partial \bigcup_{q \in\left(q_{-}, q_{+}\right)} \sigma\left(H_{p e r}+q \cdot \sum_{k} f(\cdot-k)\right)
$$

which is a subset of $\Sigma$ since $g$ is supported near $q_{-}$and $q_{+}$. Our Theorem 1.2 then holds with $\partial \Sigma$ replaced by $\partial_{\text {fluct }} \Sigma$ which can be easily seen from the proof. Note that this includes the case of closing gaps, since those appear in $\partial_{\text {fluct }} \Sigma$ by use of the open interval $\left(q_{-}, q_{+}\right)$.

(v) $p=\frac{d}{2}$ for $d \geq 5$ :

As a general assumption concerning the potentials involved we posed $V \in L_{\text {loc }}^{p}$ for $p>\frac{d}{2}$ and $d \geq 4$. The reason is that this implies that the potentials encountered are in the Kato class. It is easy to see that all the necessary background concerning deterministic Schrödinger operator is available for the limiting case $p=\frac{d}{2}$ for $d \geq 5$, since those potentials are operator small with respect to $-\Delta$ (cf. [27]). In particular, for the Combes-Thomas estimates and the trace estimates needed in Section 6 it is not hard to see that the limiting case can be included.

\section{Comparison with other results on band edge localization:}

Apart from results for Landau hamiltonians (see the references in [1]) we want to mention mainly the recent papers [10], [11] and [1]:

While presenting their results for acoustic and electromagnetic models, the methods used by Figotin and Klein in [10] and [11] also apply to random perturbations of periodic Schrödinger operators and lead to a result like our Theorem 1.1, see Remark 10 in [10]. However, their methods of proving the Wegner estimate and the initial length scale estimate require the assumption (in the Schrödinger case)

$$
0<U_{-} \leq \sum_{k \in Z^{d}} f(x-k) \leq U_{+}<\infty \text { for a.e. } x \in \mathbf{R}^{d}
$$

on the single site potential $f$ and also boundedness of $V_{\text {per }}$. This excludes unbounded $f$ and $f$ 's with small support as allowed by our Assumption A.

In [1] Barbaroux, Combes and Hislop provide a very general and powerful framework for band edge localization. This work includes periodic background potentials as a special case. However, in the periodic case it does not cover our results in full generality. In particular, their general result requires a condition ((H9) in [1]) which needs to be verified in concrete cases. In Proposition 6.4 of [1] and the remark following its proof this is done for the case of large disorder, i.e. that the support of the distribution density $g$ is $\mathbf{R}$ or at least large. In the case of a periodic background our Theorem 2.2 provides the equivalent of condition (H9) in [1] for arbitrary, possibly small, disorder. Furthermore, the proof of the Wegner estimate in [1] is given for bounded single site potential while we have included singularities. Also the localization result of [1] needs $\tau>3 d / 2$ rather then $\tau>d / 2$ in the decay assumption (1.2). The reason for this is that in addition to the improved Combes-Thomas method of [1] we also use an improved multiscale analysis based on the de Branges result. In [1] the assumption $\tau>d$ is used in a similar context and it is noted in Remark 11 that $\tau>d / 2$ would be sufficient if the improved Combes-Thomas method would be exploited. 
An important advantage of [1] is that a "linear" version of the Wegner estimate (3.1) is proven, i.e. the term $|\Lambda|^{2}$ is replaced by $|\Lambda|$. This improved form of the Wegner estimate is not needed in the proof of localization and is found at considerably higher effort than our proof of Theorem 3.1. It allows, however, to show Lipshitz continuity for the integrated density of states.

\section{APPENDIX: Proof of Lemma 5.5}

Here we give a proof of Lemma 5.5. In order to do this we first give a result for the resolvent of deterministic Schrödinger operators with a spectral gap and then apply it to the random operators $H_{\ell}(\omega)$ studied in Section 5. The deterministic result is an improved Combes-Thomas-type estimate, which differs from the 'classical' estimate by yielding a better exponential decay rate for localized resolvents at fixed energy $E$. Basically the rate is found to be proportional to the square root of the distance of $E$ to the spectrum, while the original method only gave a rate proportional to the distance. This improvement was first used in [1, Ch.3], from where we also take all essential parts of the proof. The main difference in the result and proof given below is that we need to apply the method to complex energies $E+i \varepsilon$. Some other steps in the proof are taken from a version of 'classical Combes-Thomas' in [32, Lemma 3.1].

Let $V$ be a potential on $\mathrm{R}^{d}$ which is locally uniformly in $L^{p}$ with $p$ as in assumption A of Section 1. Let $\Lambda=\Lambda_{\ell}(i)$ for some $\ell \in \mathrm{N}$ and $i \in \mathrm{Z}^{d}, H=(-\Delta+V)_{\Lambda}$ in $L^{2}(\Lambda)$ with periodic boundary conditions, and let $(r, s)$ be a spectral gap of $H$. Also let $\delta_{0}>0, \chi$, $\bar{\chi} \in L^{\infty}(\Lambda)$ with $\|\chi\|_{\infty} \leq 1,\|\tilde{\chi}\|_{\infty} \leq 1, \operatorname{dist}(\operatorname{supp} \chi, \partial \Lambda) \geq \delta_{0}, \operatorname{dist}(\operatorname{supp} \tilde{\chi}, \partial \Lambda) \geq \delta_{0}$, and $\delta:=\operatorname{dist}(\operatorname{supp} \chi, \operatorname{supp} \bar{\chi}) \geq 1$.

LFMMA A.1 Under the above assumptions there exist positive constants $C_{0}, C_{1}$ and $C_{2}$ only depending on $\delta_{0}$ and $\sup _{x}\|V\|_{L^{p}\left(\Lambda_{1}(x)\right)}$ auch that for every $E \in(r, s), \varepsilon>0$ and $i \in\{1, \ldots, d\}$ the following estimates hold:

$$
\left\|\tilde{\chi}(H-E-\mathrm{i} \varepsilon)^{-1} \chi\right\| \leq \frac{C_{2}\left(C_{0}+s\right)}{\eta} \exp \left(-\frac{C_{1}(s-r)^{1 / 2}}{C_{0}+s} \eta^{1 / 2} \delta\right)
$$

and

$$
\left\|\tilde{\chi} \partial_{i}(H-E-\mathrm{i} \varepsilon)^{-1} \chi\right\| \leq \frac{C_{2}\left(C_{0}+s\right)}{\eta} \exp \left(-\frac{C_{1}(s-r)^{1 / 2}}{C_{0}+s} \eta^{1 / 2} \delta\right)
$$

where $\eta=\operatorname{dist}(E, \sigma(H))$.

Proof. We will need a smooth function measuring the distance to supp $\chi$. For this we use the regularized distance (see $[30$, p.170f $]$ ):

There exist constants $c_{1}>0, c_{2}>0$ and $B_{\alpha}$ such that every closed set $F \subset \mathrm{R}^{d}$ admits a $C^{\infty}$-function $\Theta_{F}: \mathbf{R}^{d} \backslash F \rightarrow(0, \infty)$ with the following properties:

$$
\begin{gathered}
c_{1} \operatorname{dist}(x, F) \leq \Theta_{F}(x) \leq c_{2} \operatorname{dist}(x, F) \quad \text { for } x \in \mathrm{R}^{d} \backslash F, \\
\left|\partial^{\alpha} \Theta_{F}(x)\right| \leq B_{\alpha}(\operatorname{dist}(x, F))^{1-|\alpha|} \text { for } x \in \mathrm{R}^{d} \backslash F
\end{gathered}
$$

The constants $c_{1}, c_{2}$ and $B_{\alpha}$ do not depend on $F$ !

Fix a $C^{\infty}$-function $u: \mathrm{R} \rightarrow \mathrm{R}$ such that $u(x)=0$ for $x \leq 1 / 2$ and $u(x)=x$ for $x \geq 1$. Also choose $\varphi \in C_{0}^{\infty}(\Lambda)$ such that $\varphi(x)=1$ for $\operatorname{dist}(x, \partial \Lambda) \geq \delta_{0}, \varphi(x)=0$ for $\operatorname{dist}(x, \partial \Lambda) \leq \delta_{0} / 2$, and $\|\nabla \varphi\|_{\infty}$ is bounded by a constant only depending on $\delta_{0}$. With $F=\operatorname{supp} \chi$ define $\rho \in C_{0}^{\infty}(\Lambda)$ by $\rho(x)=\varphi(x) u\left(\Theta_{F}(x) / c_{1}\right)$ for $x \in \Lambda \backslash F$ and $\rho(x)=0$ 
for $x \in F$. One gets $\|\nabla \rho\|_{\infty} \leq C$ with a bound only depending on $c_{1}, c_{2}, B_{\alpha}$ and $\delta_{0}$, but not on $F$.

For $0<\beta \leq 1$ a calculation shows that

$$
\mathrm{e}^{\beta \rho}(H-E-\mathrm{i} \varepsilon) \mathrm{e}^{-\beta \rho}=\tilde{H}-E-\mathrm{i} \varepsilon+\mathrm{i} \beta W
$$

on $D(H)$, where $\tilde{H}:=H-\beta^{2}|\nabla \rho|^{2}$ and $W:=(-\mathrm{i} \nabla) \cdot(\nabla \rho)+(\nabla \rho) \cdot(-i \nabla)$. Note here that $D(\tilde{H})=D(H) \subset D(W)$ and that $D(H)$ is invariant under multiplication by $\mathrm{e}^{-\beta \rho}$.

The discussion of relative operator bounds in Section 1 and the fact that this implies form bounds [26, Theorem X.18] shows that there is a constant $C_{0}>-s$ only depending on $\sup _{x}\|V\|_{L^{p}\left(\Lambda_{1}(x)\right)}$ such that $\tilde{H}+C_{0} \geq 1$ and $\left\|\left(\tilde{H}+C_{0}\right)^{-1 / 2} W\left(\tilde{H}+C_{0}\right)^{-1 / 2}\right\|<1$. It follows that for every $w \in D(\tilde{H})$

$$
\|(\tilde{H}-E-\mathrm{i} \varepsilon+\mathrm{i} \beta W) w\| \geq\left\|(A+\mathrm{i} \beta B)\left(\tilde{H}+C_{0}\right)^{1 / 2} w\right\|,
$$

with the bounded operators

$$
A=\left(\tilde{H}+C_{0}\right)^{-1}(\tilde{H}-E-\mathrm{i} \varepsilon)
$$

and

$$
B=\left(\tilde{H}+C_{0}\right)^{-1 / 2} W\left(\tilde{H}+C_{0}\right)^{-1 / 2} .
$$

There is a constant $c^{\prime}>0$ only depending on $|\nabla \rho|_{\infty}$ such that for $\beta \leq c^{\prime} \eta^{1 / 2}$ one has $\operatorname{dist}(E, \sigma(\tilde{H})) \geq \eta / 2$. For such $\beta$ it follows that $0 \notin \sigma(\operatorname{Re} A)$ since $\operatorname{Re} A=(\tilde{H}+$ $\left.C_{0}\right)^{-1}(\tilde{H}-E)$.

Let $d_{+}:=\operatorname{dist}(0, \sigma(\operatorname{Re} A) \cap(0, \infty)), d_{-}:=\operatorname{dist}(0, \sigma(\operatorname{Re} A) \cap(-\infty, 0))$, and let $P_{ \pm}(\operatorname{Re} A)$ be the spectral projections on $(0, \infty)$ respectively $(-\infty, 0)$ for $\operatorname{Re} A$.

$B$ is symmetric, thus for $u \in L^{2}(\Lambda)$ and $u_{ \pm}:=P_{ \pm}(\operatorname{Re} A) u$ a calculation leads to

$$
\begin{aligned}
\|u\|\|(A+\mathrm{i} \beta B) u\| & \geq \operatorname{Re}\left\langle\left(u_{+}-u_{-}\right),(A+\mathrm{i} \beta B)\left(u_{+}+u_{-}\right)\right\rangle \\
& \geq d_{+}\left\|u_{+}\right\|^{2}+d_{-}\left\|u_{-}\right\|^{2}-2 \operatorname{Im}\left\langle u_{+},(\beta B+\operatorname{Im} A) u_{-}\right\rangle \\
& =d_{+}\left\|u_{+}\right\|^{2}+d_{-}\left\|u_{-}\right\|^{2}-2 \operatorname{Im}\left\langle u_{+}, \beta B u_{-}\right\rangle,
\end{aligned}
$$

where at the end it was used that $\operatorname{Im} A=-\varepsilon\left(\tilde{H}+C_{0}\right)^{-1}$ commutes with $P_{ \pm}(\operatorname{Re} A)$.

$\tilde{H}$ has the spectral gap $(r, s-\eta / 2)$. Thus

$$
d_{-} \geq \frac{E-r}{C_{0}+s} \geq \frac{\eta}{C_{0}+s}
$$

and

$$
d_{+} \geq \frac{s-\eta / 2-E}{C_{0}+s} \geq \frac{\eta / 2}{C_{0}+s} \geq \frac{s-E}{2\left(C_{0}+s\right)}
$$

It follows that

$$
d_{+} d_{-} \geq \frac{(E-r)(s-E)}{2\left(C_{0}+s\right)^{2}} \geq \frac{\eta(s-r)}{4\left(C_{0}+s\right)^{2}}
$$

Thus, if

$$
\beta \leq \frac{c^{\prime} \sqrt{s-r}}{4\left(C_{0}+s\right)} \eta^{1 / 2}
$$


and therefore $\beta \leq \min \left\{c^{\prime}, \frac{\sqrt{s-r}}{4\left(C_{0}+8\right)}\right\} \eta^{1 / 2}$ it follows that $\beta \leq \frac{1}{2} \sqrt{d_{+} d_{-}}$. Using that $\|B\|<1$ we get for such $\beta$ that

$$
\left|2 \operatorname{Im}\left\langle u_{+}, \beta B u_{-}\right\rangle\right| \leq \frac{1}{2} d_{+}\left\|u_{+}\right\|^{2}+\frac{1}{2} d_{-}\left\|u_{-}\right\|^{2} .
$$

Therefore (A.5), (A.6) and (A.7) imply

$$
\|u\|\|(A+\mathrm{i} \beta B) u\| \geq \frac{1}{2} d_{+}\left\|u_{+}\right\|^{2}+\frac{1}{2} d_{-}\left\|u_{-}\right\|^{2} \geq \frac{\eta}{4\left(C_{0}+s\right)}\|u\|^{2},
$$

i.e.

$$
\|(A+\mathrm{i} \beta B) u\| \geq \frac{\eta}{4\left(C_{0}+s\right)}\|u\| .
$$

Inserting this with $u=\left(\tilde{H}+C_{0}\right)^{1 / 2} w$ in (A.3) gives the conclusion that $\tilde{H}-E-\mathrm{i} \varepsilon-\mathrm{i} \beta W$ is invertible and

$$
\left\|\left(\tilde{H}+C_{0}\right)^{1 / 2}(\tilde{H}-E-\mathrm{i} \varepsilon+\mathrm{i} \beta W)^{-1}\right\| \leq \frac{4\left(C_{0}+s\right)}{\eta}
$$

for $\beta$ as in (A.8). From (A.3) it follows that for the same $\beta$

$$
\left\|\mathrm{e}^{\beta \rho}(H-E-\mathrm{i} \varepsilon)^{-1} \mathrm{e}^{-\beta \rho}\right\| \leq \frac{4\left(C_{0}+\rho\right)}{\eta}
$$

and

$$
\begin{aligned}
& \left\|\mathrm{e}^{\beta \rho} \partial_{i}(H-E-\mathrm{i} \varepsilon)^{-1} \mathrm{e}^{-\beta \rho}\right\| \\
& \leq\left\|\partial_{i}\left(\tilde{H}+C_{0}\right)^{-1 / 2}\right\|\left\|\left(\tilde{H}+C_{0}\right)^{1 / 2}(\tilde{H}-E+\mathrm{i} \beta W-\mathrm{i} \varepsilon)^{-1}\right\| \\
& \quad+\mid \beta\left(\partial_{i} \rho\right)_{\infty}\|\| \mathrm{e}^{\beta \rho}(H-E-\mathrm{i} \varepsilon)^{-1} \mathrm{e}^{-\beta \rho} \| \\
& \leq \frac{C\left(C_{0}+s\right)}{\eta} .
\end{aligned}
$$

(A1) follows by choosing $\beta=\frac{c^{\prime} \sqrt{r-8}}{4\left(C_{0}+8\right)} \eta^{1 / 2}$ and using (A.9) in

$$
\begin{aligned}
\left\|\tilde{\chi}(H-E-\mathrm{i} \varepsilon)^{-1} \chi\right\| & \leq\left\|\tilde{\chi} \mathrm{e}^{-\beta \rho}\right\|\left\|\mathrm{e}^{\beta \rho}(H-E-\mathrm{i} \varepsilon)^{-1} \mathrm{e}^{-\beta \rho}\right\|\left\|\mathrm{e}^{\beta \rho} \chi\right\| \\
& \leq \frac{C\left(C_{0}+s\right)}{\eta} \mathrm{e}^{-\beta \delta} .
\end{aligned}
$$

Here we finally used that $\Theta_{F}(x) \geq c_{1} \operatorname{dist}(x, F) \geq c_{1} \delta$ for $x \in \operatorname{supp} \tilde{\chi}$ and $\delta \geq 1$. (A.2) follows in the same way from (A.10).

We can now prove Lemma 5.5 from Lemma A.1. The expression $\| W\left(\varphi_{\ell}\right) R_{\ell}(E+$ $\mathrm{i} \varepsilon) \chi_{\ell / 3} \|$ is estimated by a sum of terms as in (A.1) or (A.2) with $H=H_{\varepsilon}\left(\omega^{\prime}\right)$ and coefficients bounded uniformly in $\ell$. The $\delta_{0}$ required in Lemma A.1 coincides with the $\delta_{0}$ of Section 5 and the functions appearing for $\chi$ and $\tilde{\chi}$ all satisfy $\delta:=\operatorname{dist}(\operatorname{supp} \chi, \operatorname{supp} \tilde{\chi}) \geq$ $\ell / 6 \geq 1$ for $\ell$ sufficiently large. If $a \in \partial \Sigma$ is a lower band edge, then we choose $(r, s):=\left(\sup \{\Sigma \cap(-\infty, a)\}, a+\ell^{\beta-2}\right)$. Since $\sigma\left(H_{\ell}(\omega)\right) \cap(-\infty, a) \subset \Sigma \cap(-\infty, a)$ it follows from Proposition 4.1 that $(r, s)$ is a spectral gap of $H_{\ell}(\omega)$ for $\omega \in \Omega_{0}$ with $\mathbf{P}\left(\Omega_{0}\right) \geq 1-\ell^{-\xi}$. (A more direct argument using Proposition 4.2 applies for $a=\inf \Sigma$ under the assumptions of Theorem 1.2.) 
For every $\omega \in \Omega_{0}$ and $E \in I\left(a-\frac{1}{2} \ell^{\beta-2}, a+\frac{1}{2} \ell^{\beta-2}\right)$ one has $\eta=\operatorname{dist}\left(E, \sigma\left(H_{\ell}(\omega)\right)\right) \geq$ $\frac{1}{2} \ell^{\beta-2}$. Inserting all this in the estimates found from (A.1) and (A.2) one gets that

$$
\sup _{\ell \neq 0}\left\|W\left(\varphi_{\ell}\right) R_{\ell}(E+\mathrm{i} \varepsilon) \chi_{\ell / 3}\right\| \leq \frac{c_{1}}{\eta} \mathrm{e}^{-c_{2} \eta^{1 / 2} \delta} \leq c_{3} \ell^{2-\beta} \mathrm{e}^{-c_{4} \ell^{\beta / 2}}
$$

with positive constants which all can be chosen uniformly in $\omega \in \Omega_{0}$ and $\ell$ because of the uniform local $L^{p}$-bounds of the random potential. If $0<\tilde{\beta}<\beta / 2$ and $\ell \geq \ell^{*}$ with $\ell^{*}$ sufficiently large, then the r.h.s. can be estimated by $\exp \left(-\ell^{\bar{\beta}}\right)$. A similar argument holds when $a \in \partial \Sigma$ is an upper band edge, which completes the proof of Lemma 5.5.

Acknowledgement. It is the pleasure of P.S. to acknowledge stays at RuhrUniversität Bochum and UAB, Birmingham during which some of the work presented here was started respectively finished. In particular, he would like to thank for the hospitality at those places as well as for the financial support by the SFB (Bochum), EPSCoR of Alabama and the DFG. Moreover, we would like to thank J.M. Combes and P.D. Hislop for valuable discussions and A. Klein for helpful comments on an earlier version of this work.

\section{REFERENCES}

1. J. M. Barbaroux, J. M. Combes and P. D. Hislop. Localization near band edges for random Schrödinger operators, Helv. Phys. Acta, 70, 16-43 (1997).

2. J. M. Combes and P. D. Hislop. Localization for some continuous, random Hamiltonians in d-dimensions, J. Funct. Anal., 124, 149-180 (1994).

3. J. M. Combes, P. D. Hislop and E. Mourre. Spectral Averaging, Perturbation of Singular Spectrum, and Localization, Trans. Amer. Math. Soc. 348, 4883-4894 (1996).

4. H. L. Cycon, R. Froese, W. Kirsch and B. Simon. Schrödinger operators. Springer Verlag, Berlin (1987).

5. L. de Branges. Perturbations of self-adjoint transformations, Am. J. Math. 84, 543-560 (1962).

6. R. del Rio, S. Jitomirskaya, Y. Last and B. Simon. Operators with singular continuous spectrum, IV. Hausdorff dimensions, rank one perturbations, and localization, J. d'Analyse Math. 69, 153-200 (1996) and R. del Rio, S. Jitomirskaya, Y. Last and B. Simon. What is localization? Phys. Rev. Lett. 75, 117-119 (1995) .

7. H. von Dreifus and A. Klein. A new proof of localization in the Anderson tight binding model, Commun. Math. Phys. 124, 285-299 (1989).

8. M. S. P. Eastham. The spectral theory of periodic differential equations. Scottish Academic Press, Edinburgh (1973).

9. A. Figotin and A. Klein. Localization phenomenon in gaps of the spectrum of random lattice operators, J. Stat. Phys. 75, 997-1021 (1994).

10. A. Figotin and A. Klein. Localization of classical waves I. Acoustic waves. Commun. Math. Phys. 180, 439-482 (1996).

11. A. Figotin and A. Klein. Localization of classical waves II. Electromagnetic Waves, Commun. Math. Phys., to appear.

12. D. Jerison and C. Kenig. Unique continuation and absence of positive eigenvalues for Schrödinger operators, Ann. of Math. 121, 463-488 (1985).

13. T. Kato. Perturbation Theory for Linear Operators, 2nd ed. Springer-Verlag, Berlin (1976). 
14. W. Kirsch. Wegner estimates and Anderson localization for alloy-type potentials, Math. Z. 221, 507-512 (1996).

15. W. Kirsch. Random Schrödinger operators. a course. In Schrödinger operators, Sonderborg DK 1988, ed. H. Holden and A. Jensen. Lecture Notes in Physics Vol. 345, Springer-Verlag, Berlin (1989).

16. W. Kirsch and F. Martinelli. On the spectrum of Schrödinger operators with a random potential, Commun. Math. Phys. 85, 329-350 (1982).

17. W. Kirsch and B. Simon. Lifshitz tails for periodic plus random potentials, J. Stat. Phys. 42, 799-808 (1986).

18. W. Kirsch, P. Stollmann and G. Stolz. Anderson localization for random Schrödinger operators with long range interaction, in preparation.

19. F. Klopp. Localization for some continuous random Schrödinger operators. Commun. Math. Phys. 167, 553-569 (1995).

20. F. Klopp. Internal Lifshitz tails for random perturbations of periodic Schrödinger operators. Preprint.

21. S. Kotani and B. Simon. Localization in general one dimensional systems, II. Continuum Schrödinger operators, Commun. Math. Phys. 112, 103-120 (1987).

22. G. A. Mezincescu. Lifshitz singularities for periodic operators plus random potentials. J. Stat. Phys. 49, 1181-1190 (1987).

23. L. Pastur and A. Figotin. Random Schrödinger operators. Springer-Verlag, Berlin (1992).

24. Th. Poerschke, G. Stolz. On eigenfunction expansions and scattering theory, Math. Z. 212, 337-357 (1993).

24. Th. Poerschke, G. Stolz, J. Weidmann. Expansions in generalized eigenfunctions of selfadjoint operators, Math. Z. 202, 397- 408 (1989).

26. M. Reed and B. Simon. Methods of Modern Mathematical Physics II. Academic Press, New York (1975).

27. M. Reed and B. Simon. Methods of Modern Mathematical Physics IV. Academic Press, New York (1978).

28. R. Regbaoui. Unicité forte pour les operateurs de Schrödinger avec potentiels de Kato. J. Funct. Anal. 134, 281-296 (1995).

29. T. Spencer. Localization for random and quasi-periodic potentials, J. Stat. Phys. 51, 1009-1019 (1988) .

30. E. M. Stein. Singular Integrals and Differentiability Properties of Functions. Princeton University Press, Princeton (1970).

31. P. Stollmann. Localization for random perturbations of anisotropic periodic media. Israel J. Math., to appear.

32. P. Stollmann, G. Stolz. Singular spectrum for multidimensional Schrödinger operators with potential barriers. J. Operator Theory 32, 91-109 (1994).

33. G. Stolz. Localization for Schrödinger operators with effective barriers. J. Funct. Anal., to appear.

34. W. Thirring. A Course in Mathematical Physics 3, Quantum Mechanics of Atoms and Molecules. Springer-Verlag, New York, Wien, (1981).

35. J. Weidmann. Linear Operators in Hilbert Spaces. Graduate Texts in Mathematics Vol.68, Springer Verlag, Berlin (1980).

36. J. Weidmann. Spectral Theory of Ordinary Differential Operators, Lecture Notes in Mathematics, Vol.1258, Springer Verlag, Berlin (1987). 